\title{
Evaluate Status of Pacific Lamprey in the Clearwater River Drainage, Idaho
}

\section{Annual Report}

2002

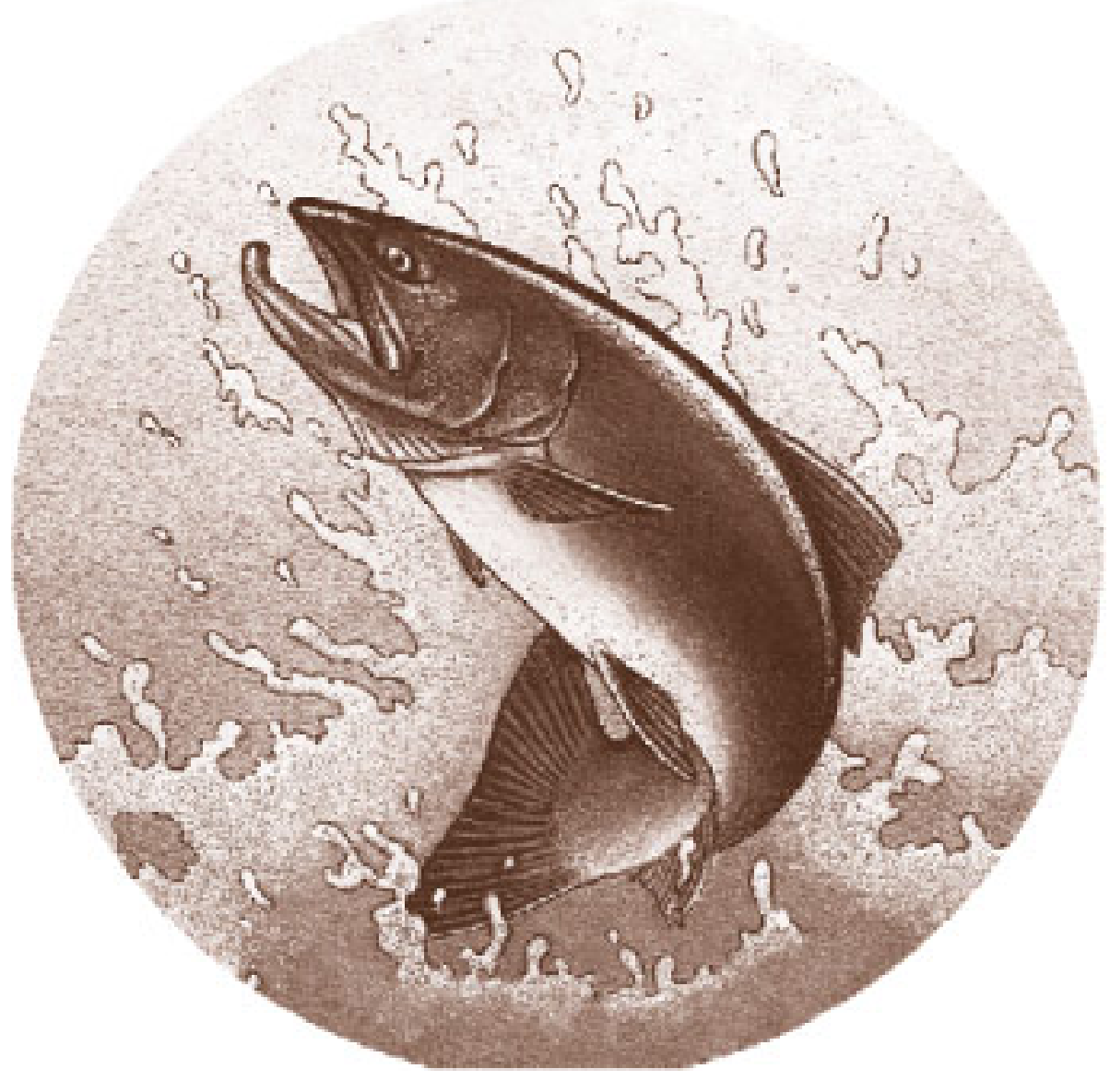


This Document should be cited as follows:

Cochnauer, Tim, Christopher Claire, "Evaluate Status of Pacific Lamprey in the Clearwater River Drainage, Idaho", 2002 Annual Report, Project No. 200002800, 48 electronic pages, (BPA Report DOE/BP-00004039-2)

\author{
Bonneville Power Administration \\ P.O. Box 3621 \\ Portland, OR 97208
}

This report was funded by the Bonneville Power Administration (BPA), U.S. Department of Energy, as part of BPA's program to protect, mitigate, and enhance fish and wildlife affected by the development and operation of hydroelectric facilities on the Columbia River and its tributaries. The views in this report are the author's and do not necessarily represent the views of BPA. 


\section{EVALUATE STATUS OF PACIFIC LAMPREY IN THE CLEARWATER RIVER DRAINAGE, IDAHO}

ANNUAL REPORT 2002

Prepared by:

Tim Cochnauer, Sr. Fishery Research Biologist

Christopher Claire, Fishery Technician

Idaho Department of Fish and Game

Clearwater Region

1540 Warner

Lewiston, ID 83501

Prepared for:

U.S. Department of Energy

Bonneville Power Administration

Environmental Fish and Wildlife

P.O. Box 3621

Portland, OR 98203-3621

Project Number 2000-028-00

Contract Number 00000090-00001 


\section{TABLE OF CONTENTS}

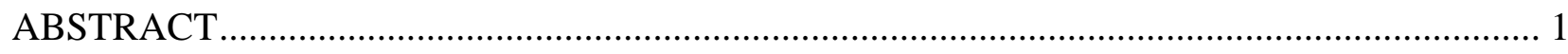

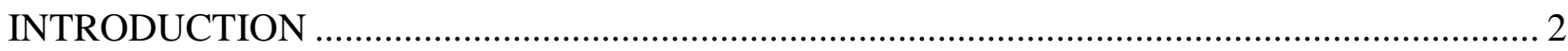

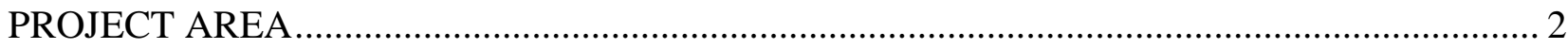

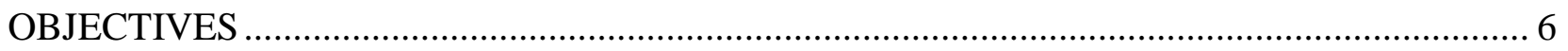

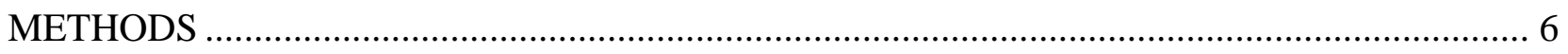

RESULTS

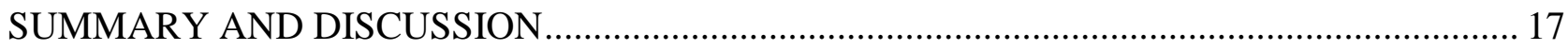

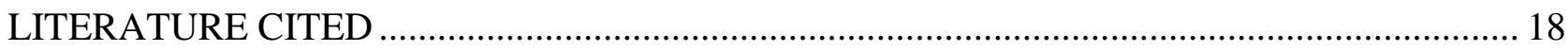

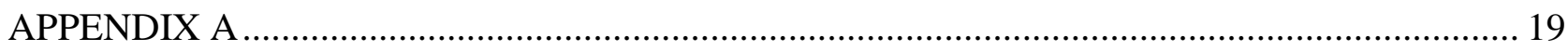

\section{LIST OF TABLES}

Table 1. Habitat type and substrate classification for sampling sites in the South Fork Clearwater River Drainage, ID................................................................... 7

Table 2. Habitat locations of Pacific lamprey ammocoetes in randomly sampled units in Red River and selected units in South Fork Clearwater River, ID,

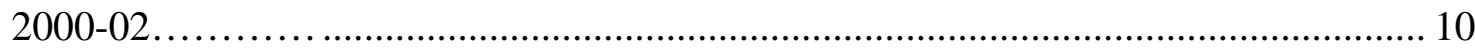

Table 3. Presence-absence surveys of Pacific lamprey ammocoetes in selected units Red River, South Fork Clearwater River, Selway River, and Lochsa River, Clearwater River drainage, ID, 2001-02.

\section{LIST OF FIGURES}

Figure 1. Geographic location of Pacific lamprey investigations in the South Fork Clearwater River, drainage, 2000-02. 4

Figure 2. Geographic location of Pacific lamprey investigations in the South Fork Clearwater River, drainage, 2000-02 . 5 


\section{LIST OF FIGURES CONT.}

Figure 3. Length frequency of Pacific lamprey $(\mathrm{N}=130)$ captured in Red River migrant trap, South Fork Clearwater River drainage, ID, 2002 ........................................... 8

Figure 4. Length frequency of Pacific lamprey $(\mathrm{N}=605)$ captured by electroshocking in Red River, ID, 2000-02

Figure 5. Length frequency of Pacific lamprey $(\mathrm{N}=447)$ captured by electroshocking in South Fork Clearwater River, ID, 2000-02.

Figure 6. Length frequency of Pacific lamprey $(\mathrm{N}=61)$ captured by electroshocking in Selway River, ID, 2000-02

Figure 7. Length frequency of Pacific lamprey $(\mathrm{N}=51)$ captured by electroshocking in Lochsa River, ID, 2000-02

Figure 8. Pacific lamprey ammocoetes and macrothalmia captured April 1-October 31, 1993-2002 in Red River trap (rkm 5.0), South Fork Clearwater River drainage, ID. 14

Figure 9. Pacific lamprey ammocoete and macrothalmia length and weight relationship ( $\mathrm{N}=333$ ), Red River, ID, 2000-01 15

Figure 10. Pacific lamprey ammocoete and macrothalmia length and weight relationship (N=301), South Fork Clearwater River, ID, 2000-01

Figure 11. Pacific lamprey ammocoete and macrothalmia adjusted bypass/collection count and Snake River flows at Lower Granite dam, WA, 1998 


\begin{abstract}
In 2002 Idaho Department of Fish and Game continued investigation into the status of Pacific lamprey populations in Idaho's Clearwater River drainage. Trapping, electrofishing, and spawning ground redd surveys were used to determine Pacific lamprey distribution, life history strategies, and habitat requirements in the South Fork Clearwater River, Lochsa River, Selway River, and Middle Fork Clearwater River subbasins. Five-hundred forty-one ammocoetes were captured electroshocking 70 sites in the South Fork Clearwater River, Lochsa River, Selway River, Middle Fork Clearwater River, Clearwater River, and their tributaries in 2002. Habitat utilization surveys in Red River support previous work indicating Pacific lamprey ammocoete densities are greater in lateral scour pool habitats compared to riffles and rapids. Presence-absence survey findings in 2002 augmented 2000 and 2001 indicating Pacific lamprey macrothalmia and ammocoetes are not numerous or widely distributed. Pacific lamprey distribution was confined to the lower reaches of Red River below rkm 8.0, the South Fork Clearwater River, Lochsa River (Ginger Creek to mouth), Selway River (Race Creek to mouth), Middle Fork Clearwater River, and the Clearwater River (downstream to Potlatch River).
\end{abstract}




\section{INTRODUCTION}

The Pacific lamprey Lampetra tridentata is facing the same migratory hazards and habitat degradation as other anadromous fish species in Idaho. Because this fish is not recognized as a sport or game fish species, little attention has been given to its status.

The ecological interaction of Snake River Pacific lamprey populations and other riverine species is thought to contribute to Snake River basin overall aquatic productivity. Pacific lamprey ammocoetes provide Snake River basin white sturgeon Acipenser transmontanus populations with an important food source which potentially contributes to Snake River white sturgeon population productivity (Galbreath 1979). Pacific lamprey adults are a source of marine derived nutrients in the Snake River basin. Aquatic and avian predator utilization of ammocoetes and macrothalmia potentially results in reduced predation impact to outmigrating juvenile salmon Oncorhynchus spp. and steelhead trout O. mykiss in the lower Snake River migrational corridor. Pacific lamprey, chinook salmon, and summer steelhead trout, rear in Snake River basin stream habitats, however, the ecological relationship interactions of the three species in the basin are little known. Basic life history, distribution, and remaining population status are urgently needed to fully understand this species and to begin intensive management before populations decline to critical, unrecoverable threshold in Idaho.

Pacific lamprey ammocoetes are eyeless upon hatching and filter feed 4-7 years before undergoing transformation into macrothalmia. Transformation changes include formation of an oral disc and eyes among other morphological processes. Following transformation Pacific lamprey migrate to the ocean and parasitically feed on a wide range of marine aquatic organisms (Scott and Crossman 1973) for an estimated 1-2 years prior to returning to freshwater to spawn. The Clearwater River drainage of north central Idaho is an important study area as both Pacific lamprey ammocoetes and macrothalmia have been captured in South Fork Clearwater River (S.F. CLEARWATER RIVER) anadromous fish smolt traps since 1992.

Understanding Pacific lamprey larval fish population composition, migrational behavior, and habitat needs will provide basic information to better manage Pacific lamprey. Without this knowledge, the opportunity for preservation of critical habitat may be lost. This project will add to our knowledge of Pacific lamprey and provide critical information to minimize future degradation of habitat.

\section{PROJECT AREA}

The Clearwater River drainage is located in north central Idaho and encompasses approximately 2.5 million hectares. The major tributaries include the Potlatch, S.F. Clearwater, N.F. Clearwater, M.F. Clearwater, Selway, and Lochsa rivers (Figure 1).

Hydroelectric dam construction in the Clearwater River drainage has impacted salmon, steelhead, and Pacific lamprey populations. The Pacific Power and Light 
Lewiston Dam, was constructed in 1927 at Clearwater River rkm 1.8. The dam was originally constructed with two upstream passage ladders, but obstructed steelhead trout passage somewhat and salmon passage severely. In the 1927-1940 period when the problem was detected and remedied, spring chinook salmon and fall chinook salmon populations were reduced to remnant numbers and subsequently never recovered (White 1954). The impacts of the Lewiston Dam to Pacific lamprey macrothalmia and ammocoetes downstream migrants and upstream migrating adults are unknown. Dworshak Dam, constructed in 1972 on the North Fork Clearwater River (rkm 1.8) was constructed without an upstream passage ladder. Anadromous salmon, steelhead, and Pacific lamprey populations upstream of the project are now considered extinct. Harpster Dam constructed in 1910 on the S.F. Clearwater River blocked salmon and steelhead trout upstream migration in the 1949-1963 period, however, impacts to Pacific lamprey are unknown. Steelhead trout migration was possible, although limited, over the dam from 1935 to 1949. High flows destroyed the fishway in 1949 eliminating adult salmonid passage until the dam was removed in 1963. Adult Pacific lamprey passage may have occurred during this entire period as adult Pacific lamprey have the ability to climb above water surface levels (G. Starke, U.S. Army Corps of Engineers, personal communications). Pacific lamprey returns following removal of the dam could have provided recolonization stock for the S.F. Clearwater River drainage.

The land ownership of the Clearwater River basin is U. S. Forest Service, Bureau of Land Management, and other Federal Lands (58.0\%), State of Idaho (6.0\%), Timber Company Land (8.0\%), Nez Perce Tribe (4.0\%), and Private (24.0\%). Land use in the Clearwater River drainage is primarily agricultural or livestock pasture grazing in the lower and central basin and forestry related in the headwater reaches. Mining predominantly occurred in the S.F. Clearwater River drainage.

The S.F. Clearwater River drains 300,440 hectares. The upper S.F. Clearwater River (Figure 2.) watershed has several large meadow complexes with low stream gradients and fine substrates. The mid and lower S.F. Clearwater River reaches are predominantly canyon confined and boulder substrate dominated. The current land ownership of the S.F. Clearwater River watershed is U.S. Forest Service (68\%), private (28\%), Nez Perce Tribe (0.9\%), Bureau of Land Management (2\%), and State of Idaho $(0.7 \%)$. Extensive mining from the 1860's to the mid-1900's occurred in four S.F. Clearwater River tributaries, Crooked River, Red River, American River, and Newsome Creek. 


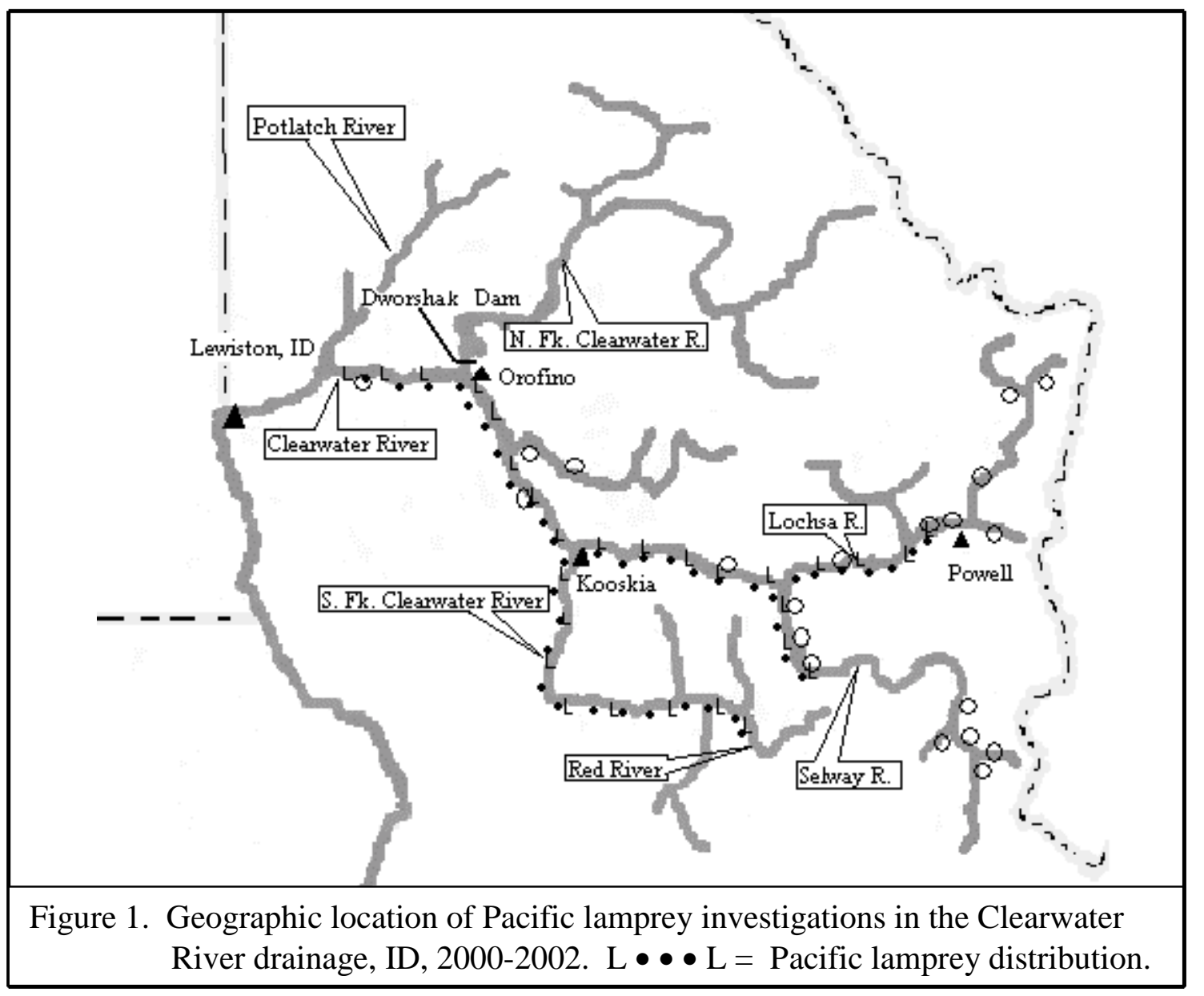




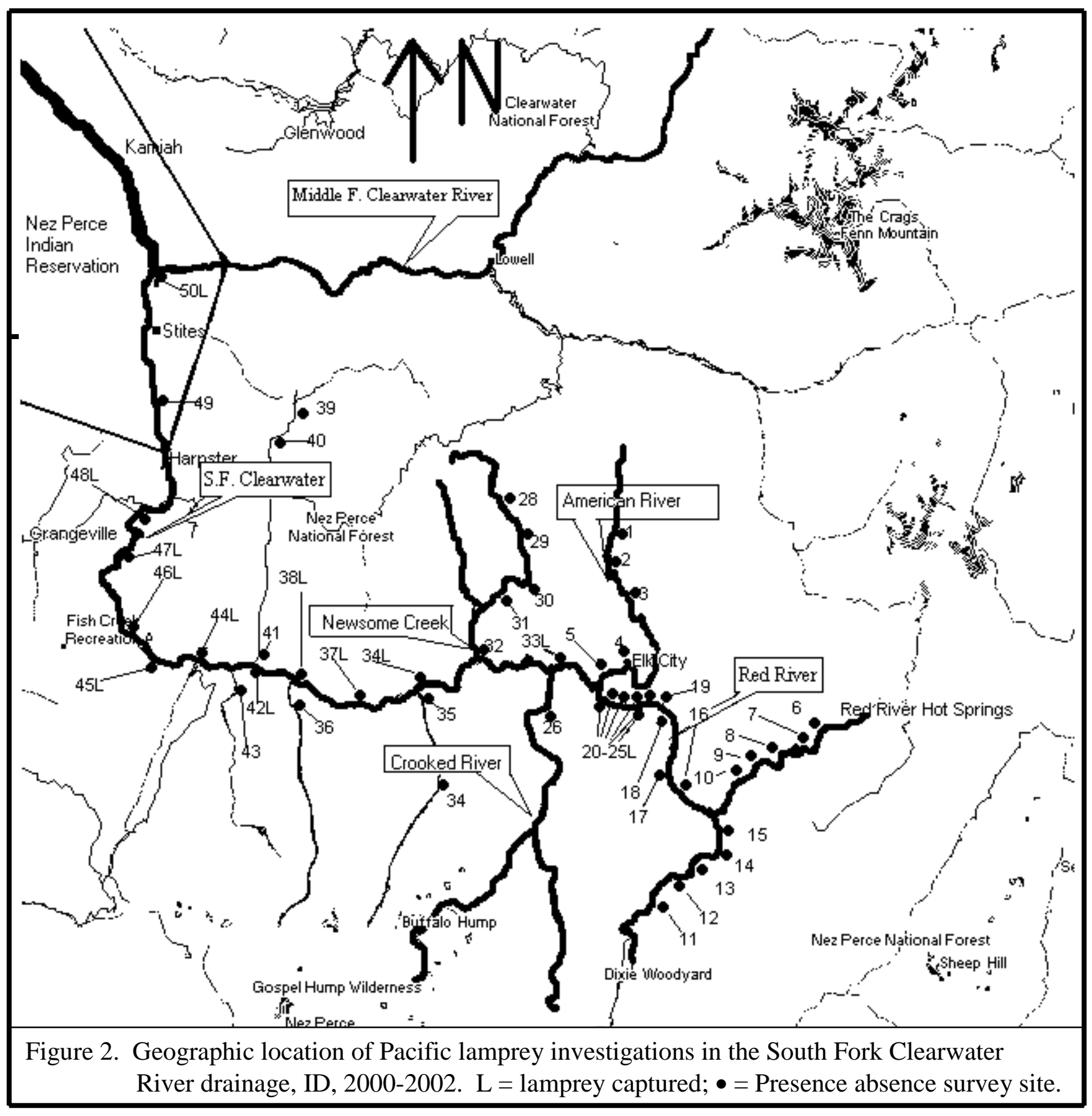




\section{OBJECTIVES}

1. Determine life history characteristics of Pacific lamprey ammocoetes and macrothalmia in the Clearwater River drainage.

2. Determine habitat requirements of Pacific lamprey in the Clearwater River drainage.

3. Determine distribution of Pacific lamprey in the Clearwater River drainage.

4. Develop and implement strategies to protect Pacific lamprey adult, juvenile, and larval habitat.

\section{METHODS}

Electroshocking with an ABP-2 electrofisher was used to capture fish in the stream channel. Any Pacific lamprey mortalities were retained for statolith banding age determination, following procedures described by (Beamish and Medland 1988). Presence-absence surveys were completed in the Red River, S.F. Clearwater River, S.F. Clearwater River tributaries, Selway River, Lochsa River and tributaries, Potlatch River, and the Middle Fork of the Clearwater River (Figure 1).

Determination of habitat usage was focused in the Red River drainage. We segmented Red River into one-kilometer sections from its mouth upstream to the uppermost bridge crossing ( $\mathrm{rkm}$ 41). We prioritized eight Red River habitat sampling locations based on random selection of kilometer section and then sampled the first 100 meters of each selected section. The habitat in each of the sampled sections was classified as to type (Table 1). The first riffle, pool, glide, etc., was electroshocked from its downstream boundary upstream without repeating sampling in identical habitat types. Water depth, water velocity, substrate composition, and canopy cover were measured and recorded at the site of individual captures.

Three downstream migrant traps currently operated by Idaho Department of Fish and Game (IDFG) in the S.F. Clearwater River drainage were used to monitor Pacific lamprey downstream movements. The Crooked River scoop trap (rkm 1.0) was replaced with a $1.5 \mathrm{~m}$ rotary screen trap in 2002 and operated from April 4 to October 31. A $1.50 \mathrm{~m}$ diameter rotary screen trap on American River (rkm 3.0) was operated from April 12 to October 31. Another 1.50 m diameter rotary screen trap on Red River (rkm 5.0) was operated from April 10 to October 31.

All captured Pacific lamprey ammocoetes and macrothalmia were anesthetized, and total lengths and body weights measured. Individuals were then recovered in fresh water and released near the site of capture. Outmigrant estimates at traps were made using (Beamish and Levings 1991) trap-area fished methods. 
Table 1. Habitat unit and substrate classification for sampling sites in the Clearwater River drainage, ID, 2000-02.

\begin{tabular}{|c|c|c|}
\hline Habitat Units & I.D. & Substrate Classification \\
\hline Falls & FLL & Substrate Type (mm) \\
\hline & & Large Boulder \\
\hline Cascades & CAS & Small Boulder 256-512 \\
\hline & & Cobble \\
\hline Rapids & & Coarse Gravel 16-64 \\
\hline Typical & RTT & Medium Gravel \\
\hline Boulders & RBB & Fine Gravel \\
\hline Bedrock & RBD & Course Sand \\
\hline & & Fine Sand $\quad 0.062-0.50$ \\
\hline Riffles & & Silt/Organic $0.004-0.062$ \\
\hline Typical & RIF & \\
\hline Pocket-water & RIP & \\
\hline Glide & GLD & \\
\hline Pools & & \\
\hline Lateral Scour Pool & LSP & \\
\hline Straight Scour Pool & SCP & \\
\hline Plunge Pool & PPP & \\
\hline Dammed Pool & DMP & \\
\hline Alcove & ALC & \\
\hline
\end{tabular}

In 2002 visual redd surveys for spawning adult Pacific lamprey were conducted from May 1 to July 1 on 0.5 kilometer of Red River and its tributaries.

Pacific lamprey ammocoete and macrothalmia collection and passage information at Lower Granite Dam was obtained from the Washington Department of Fish and Wildlife personnel to determine the magnitude and timing of Pacific lamprey downstream migration. Mortality samples of macrothalmia and ammocoetes were collected to obtain genetic material and assess the general size of juvenile and larval Pacific lamprey captured.

\section{RESULTS}

During 2002, no Pacific lamprey ammocoetes or macrothalmia were captured in the Crooked River or American River rotary screen traps. One-hundred twenty-five 
Pacific lamprey ammocoetes and 20 macrothalmia were captured in the Red River rotary screen trap. The average total length of the ammocoetes was $140 \mathrm{~mm}$ and the average total length for macrothalmia was $143 \mathrm{~mm}$ (Figure 3).

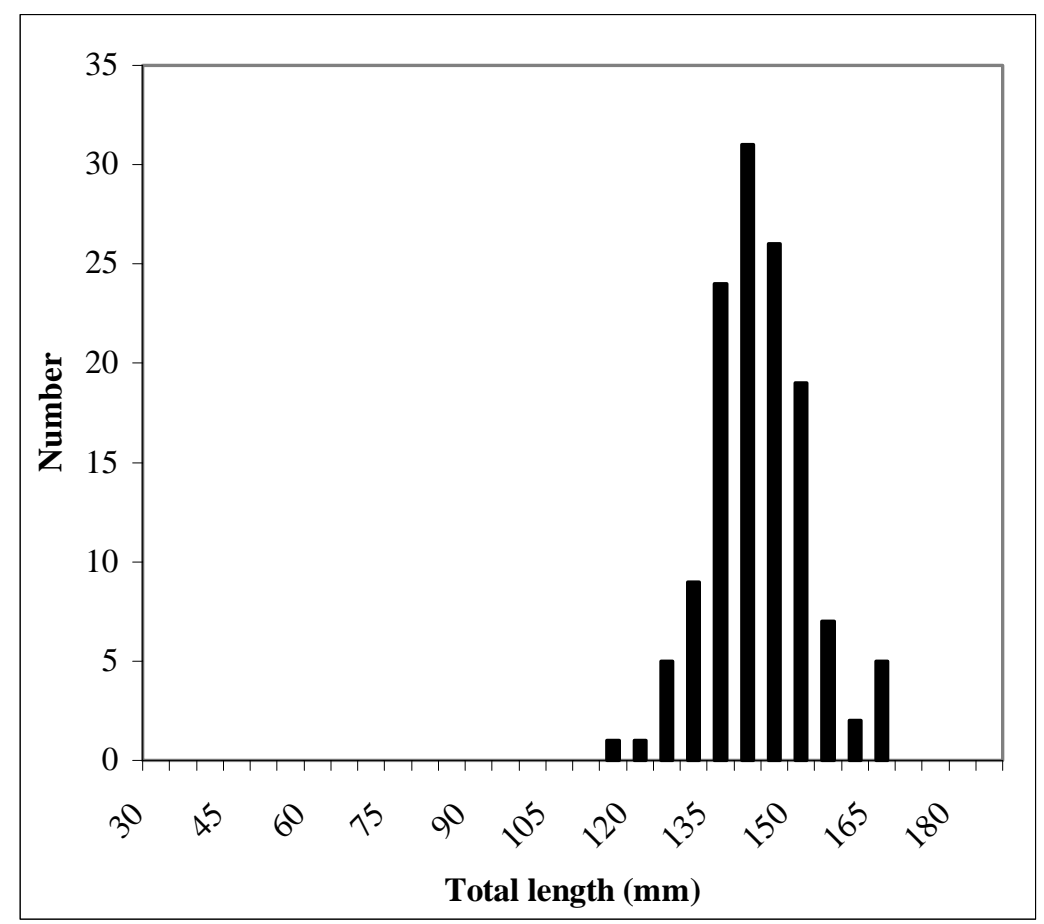

Figure 3. Total length frequency of $(\mathrm{N}=130)$ Pacific lamprey ammocoetes captured in the Red River migrant trap, ID, 2002.

Based on trap-area fished, a total of 875 ammocoetes and 140 macrothalmia were estimated to have migrated past the Red River trap in 2002, compared to 307 ammocoetes and seven macrothalmia in 2001, and 175 ammocoetes and 14 macrothalmia in 2000 .

A total of 274 Pacific lamprey ammocoetes were captured by electroshocking in Red River. No macrothalmia were captured electroshocking in Red River. Pacific lamprey ammocoetes were found in seven sections of Red River up to rkm 7.5. No Pacific lamprey ammocoetes or macrothalmia were captured in Red river sample sites at $\mathrm{rkm}$ 8.0, 9.0, and 10. The largest Pacific lamprey ammocoete captured electroshocking in the S.F. Clearwater River drainage in 2002 was 155 mm (TL) and the smallest Pacific lamprey ammocoete captured measured $77 \mathrm{~mm}$ (TL). No macrothalmia were captured electroshocking in the S.F. Clearwater River drainage in 2002.

More Pacific lamprey ammocoetes were captured in Red River sampling lateral scour pool habitat than any other single habitat type (Table 2), however, no alcove habitat was sampled in 2001 or 2002 where the greatest density of $253.3 / 100 \mathrm{~m}^{2}$ was found in 2000. Individuals were mostly found inhabiting sand and silt substrates in calm water 
sites adjacent to overhanging riparian canopy cover or in low velocity pockets behind boulders. Pacific lamprey ammocoetes were captured in water depths ranging from $0.1-$ $1.0 \mathrm{~m}$, however, they were predominantly captured in depths $<0.60 \mathrm{~m}$.

Two Pacific lamprey marked at Red River rkm 3.4 in 2000, were recaptured in 2001 within $40.0 \mathrm{~m}$ of the original capture location (Table 3). No marked Pacific lamprey ammocoetes were recaptured in 2002. The orange elastomer marks observed in 2001 retained clarity of when the ammocoetes were marked in 2000.

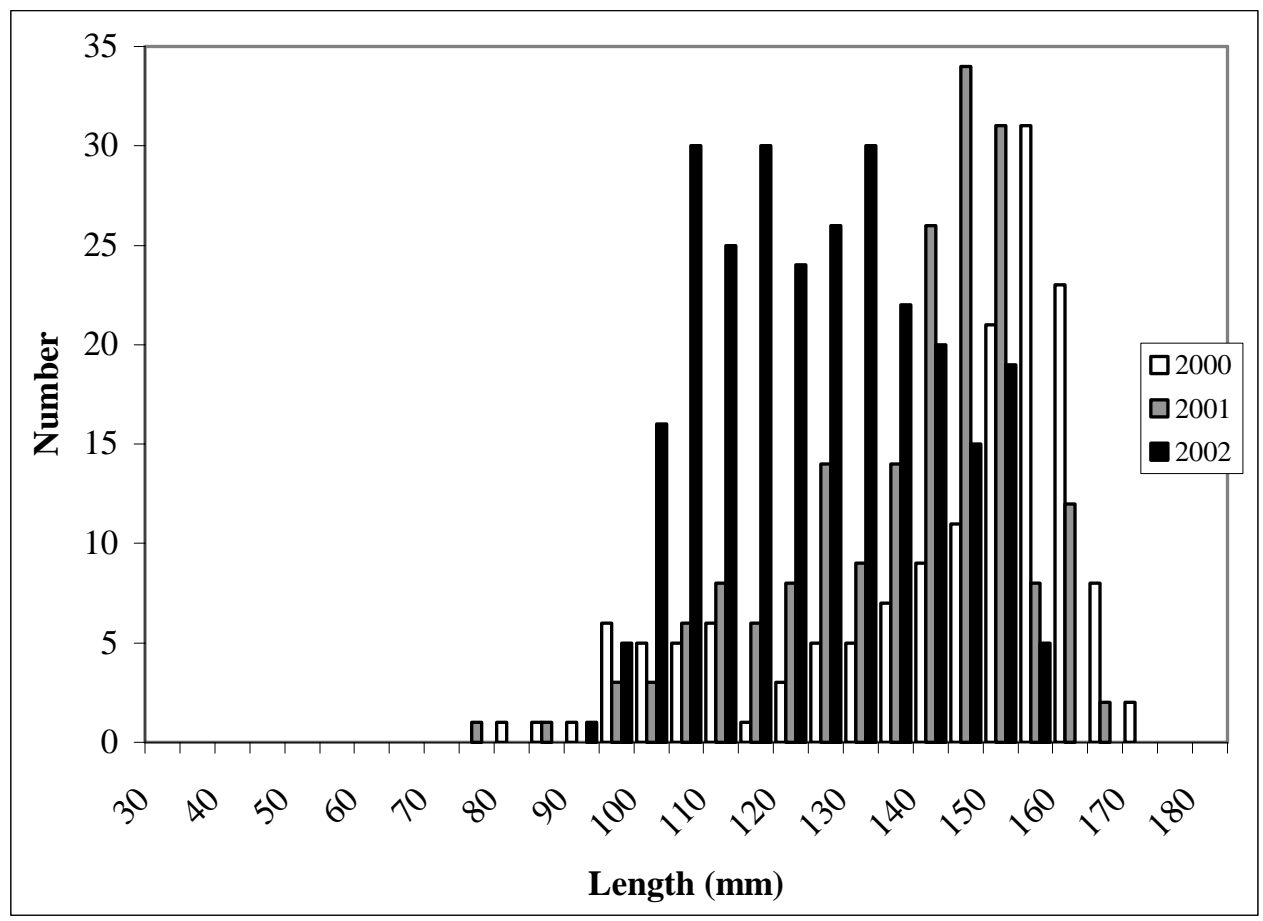

Figure 4. Total length frequency of Pacific lamprey ammocoetes and macrothalmia $(\mathrm{N}=151,2000 ; \mathrm{N}=186,2001 ; \mathrm{N}=268,2002)$ captured by electroshocking in Red River, ID, 2000-02.

Although numerous potential spawning sites were identified, no adult Pacific lamprey or redds were observed during 2002 surveys. Redd surveys were restricted to a $0.5 \mathrm{~km}$ reach at Red River rkm 43.0 due to the suspected low number of adult spawners in the S.F. Clearwater River drainage.

No Pacific lamprey were found sampling the Selway River above Whitecap Creek, Selway River tributaries, Lochsa River tributaries, M.F. Clearwater River 
tributaries, or Potlatch River. Pacific lamprey distribution in the Clearwater River drainage was limited to lower $7.5 \mathrm{~km}$ of Red River, the S.F. Clearwater River, Selway River (Race Creek to mouth), Lochsa River (Ginger Creek to mouth), M.F. Clearwater River, and the Clearwater River. One-hundred seven Pacific lamprey ammocoetes were sampled in the S.F. Clearwater River, 64 in the Selway River, and 60 in the Lochsa River (Figures 5, 6, and 7). The largest ammocoete captured in the Clearwater River drainage was captured in Red River (155 mm TL) and the smallest was captured in the Clearwater River (25 mm TL).

Table 2. Habitat locations of Pacific lamprey larvae in randomly sampled units in Red River, ID, 2000-02.

\begin{tabular}{|c|c|c|c|c|c|}
\hline Habitat Type & $\begin{array}{l}\text { Lamprey } \\
\text { Captured }\end{array}$ & $\begin{array}{c}\text { Total } \\
\text { Area Fished } \\
\mathrm{m}^{2}\end{array}$ & $\begin{array}{l}\text { Total } \\
\text { Time Fished } \\
\text { (Min) }\end{array}$ & Lamprey $/ 100 \mathrm{~m}^{2}$ & $\begin{array}{c}\text { C.P.U.E. } \\
\text { (Lamprey/Min) }\end{array}$ \\
\hline Lateral Scour Pool & 342 & 1283.4 & 1461 & 26.648 & 0.234 \\
\hline Riffle & 15 & 603.5 & 726 & 2.486 & 0.021 \\
\hline $\begin{array}{l}\text { Riffle with pockets } \\
\text { (pocket water) }\end{array}$ & 57 & 1269.8 & 825 & 4.489 & 0.069 \\
\hline Rapids with boulders & 10 & 357.3 & 305 & 2.799 & 0.033 \\
\hline $\begin{array}{l}\text { Alcove } \\
\text { (non random) }\end{array}$ & 19 & 7.5 & 20 & 253.333 & 0.950 \\
\hline TOTALS: & 443 & 3521.5 & 3337 & -- & -- \\
\hline
\end{tabular}


Table 3. Presence-absence surveys of Pacific lamprey larvae in Red River, South Fork Clearwater River, Selway River, and Lochsa River, ID, 2001-02.

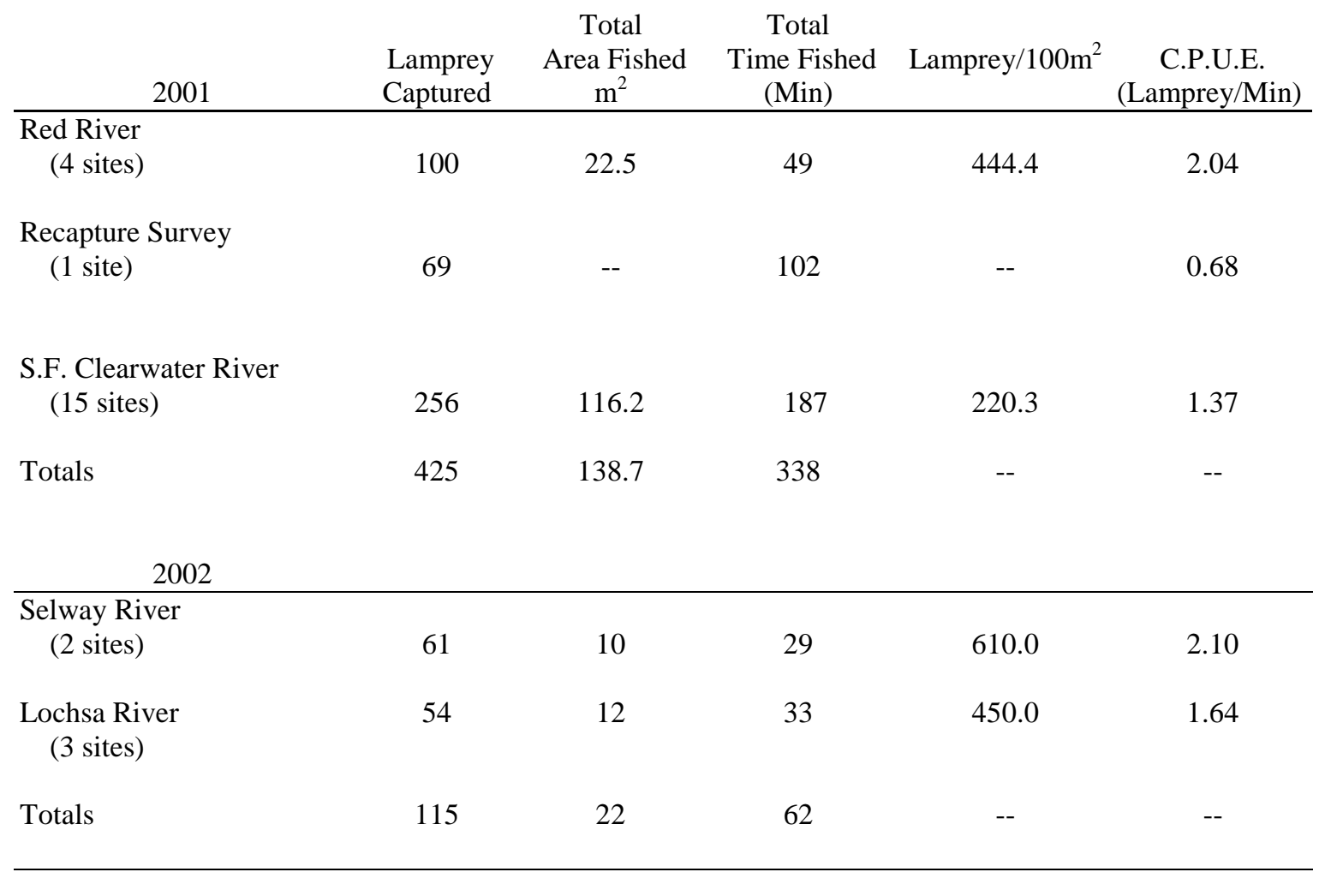




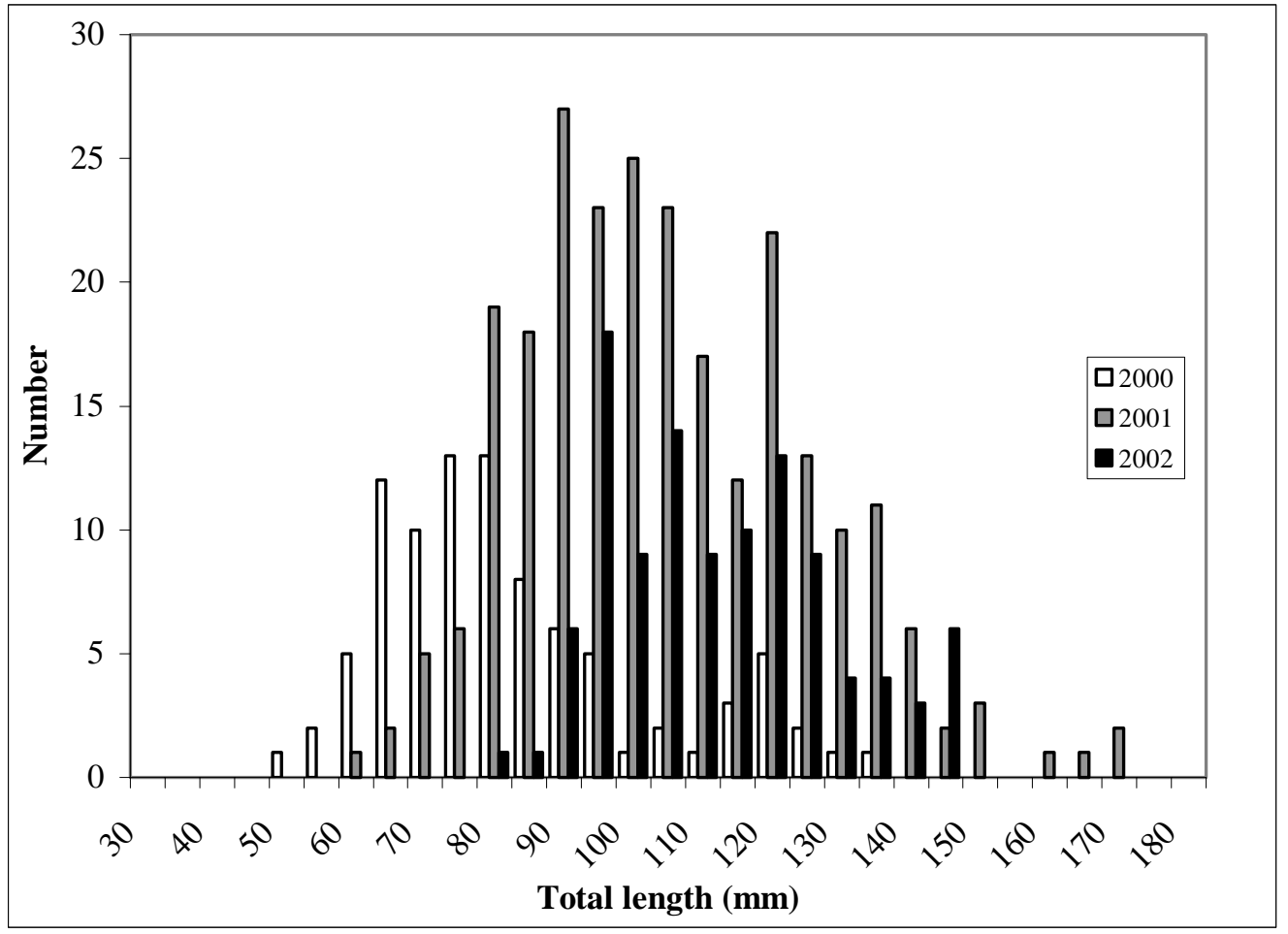

Figure 5. Total length frequency of Pacific lamprey ammocoetes and macrothalmia $(\mathrm{N}=91,2000 ; \mathrm{N}=253,2001 ; \mathrm{N}=107,2002)$ captured by electroshocking in S.F. Clearwater River, ID, 2000-02.

Downstream migration of ammocoetes and macrothalmia in Red River occurs predominantly at night from mid-March to May 31, with a limited number captured in September and October (Figure 8). Out of the total ( $N=821$ ) Pacific lamprey ammocoetes captured April 1 to October 31, 1993-2001 87\% occurred between April 1 and May 31. In 1996 a total of 25 Pacific lamprey ammocoetes were captured in the March 13 to March 31 period, which is important information indicating an unknown portion of downstream migration occurs prior to the general April 1 - October 31 trapping period. 


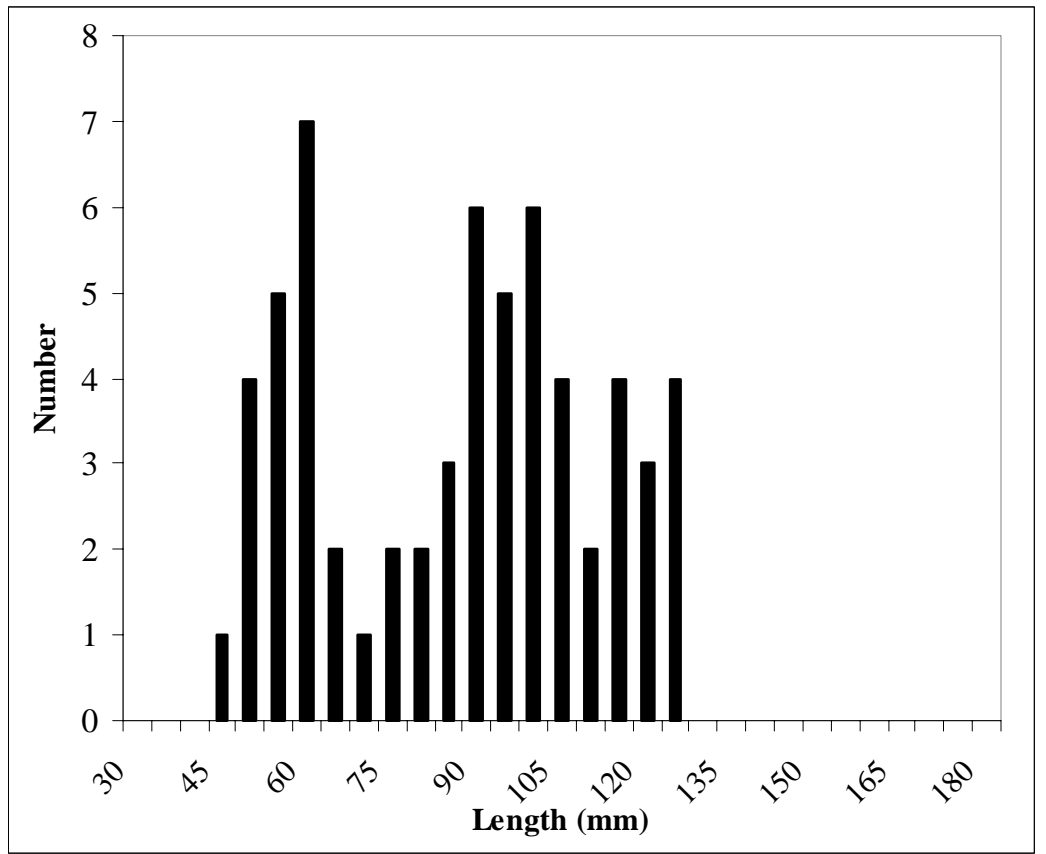

Figure 6. Total length frequency of Pacific lamprey ammocoetes $(\mathrm{N}=61)$ captured electroshocking in the Selway River, ID, 2002.

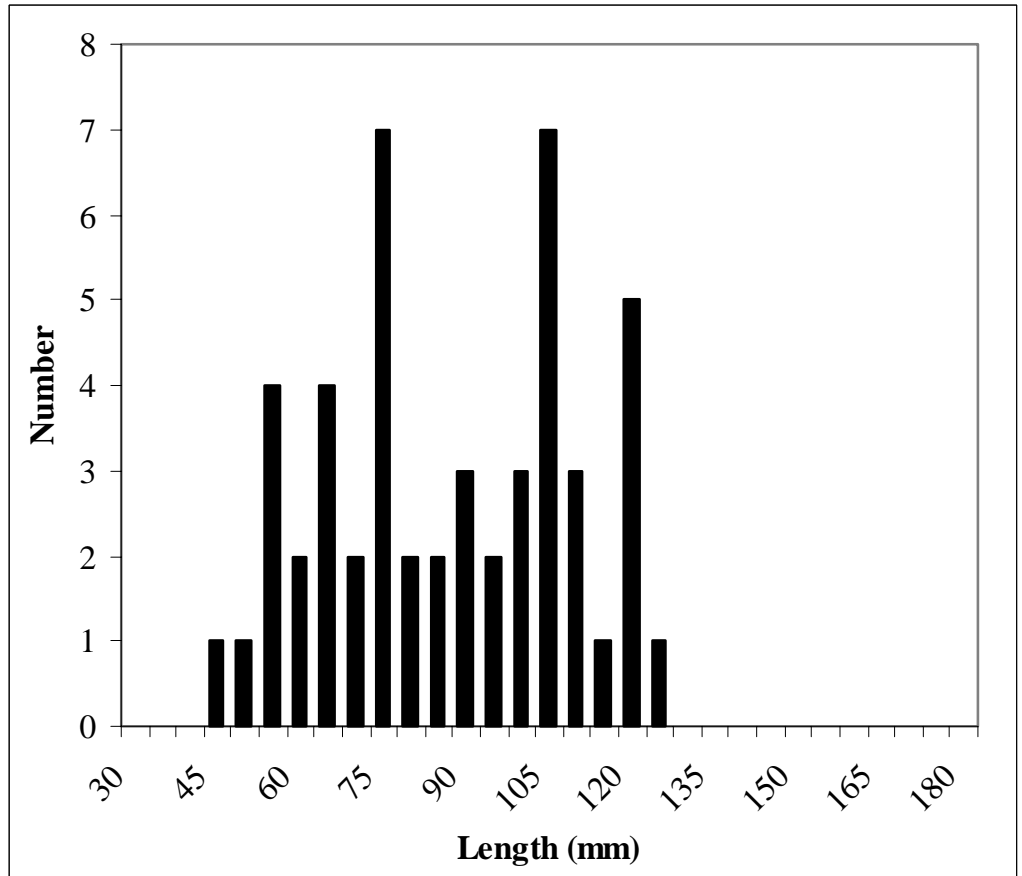

Figure 7. Total length frequency of Pacific lamprey ammocoetes $(\mathrm{N}=50)$ captured electroshocking in the Lochsa River, ID, 2002. 


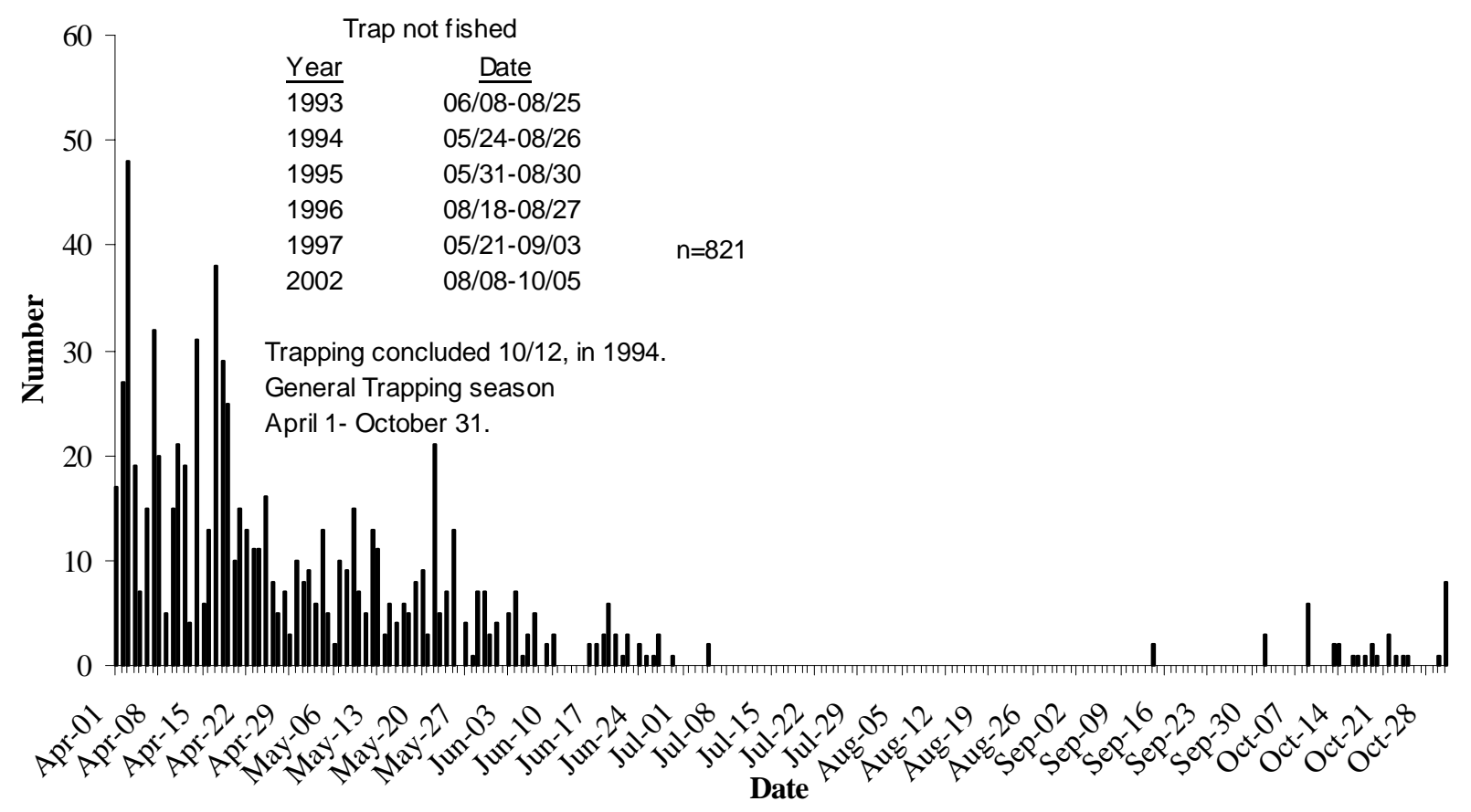

Figure 8. Pacific lamprey ammocoetes and macrothalmia captured April 1-October 31, 1993-2002 in Red River trap (rkm 5.0), ID.

The length and weight relationship of Pacific lamprey ammocoetes captured in Red River and S.F. Clearwater River indicates that weight increases exponentially with length (Figures 9 and 10). The majority of Red River Pacific lamprey ammocoetes and macrothalmia captured were greater than $120 \mathrm{~mm}$ TL, however, ammocoetes and macrothalmia electroshocked in the S.F. Clearwater River were predominantly $<120 \mathrm{~mm}$ TL (Figure 10).

Pacific lamprey juvenile and larval passage information obtained from Washington Department of Fish and Wildlife at Lower Granite Dam in 2002 supports the 2000-2001 assessment indicating that the majority of ammocoetes and macrothalmia pass the project between March 1 and July 1. 


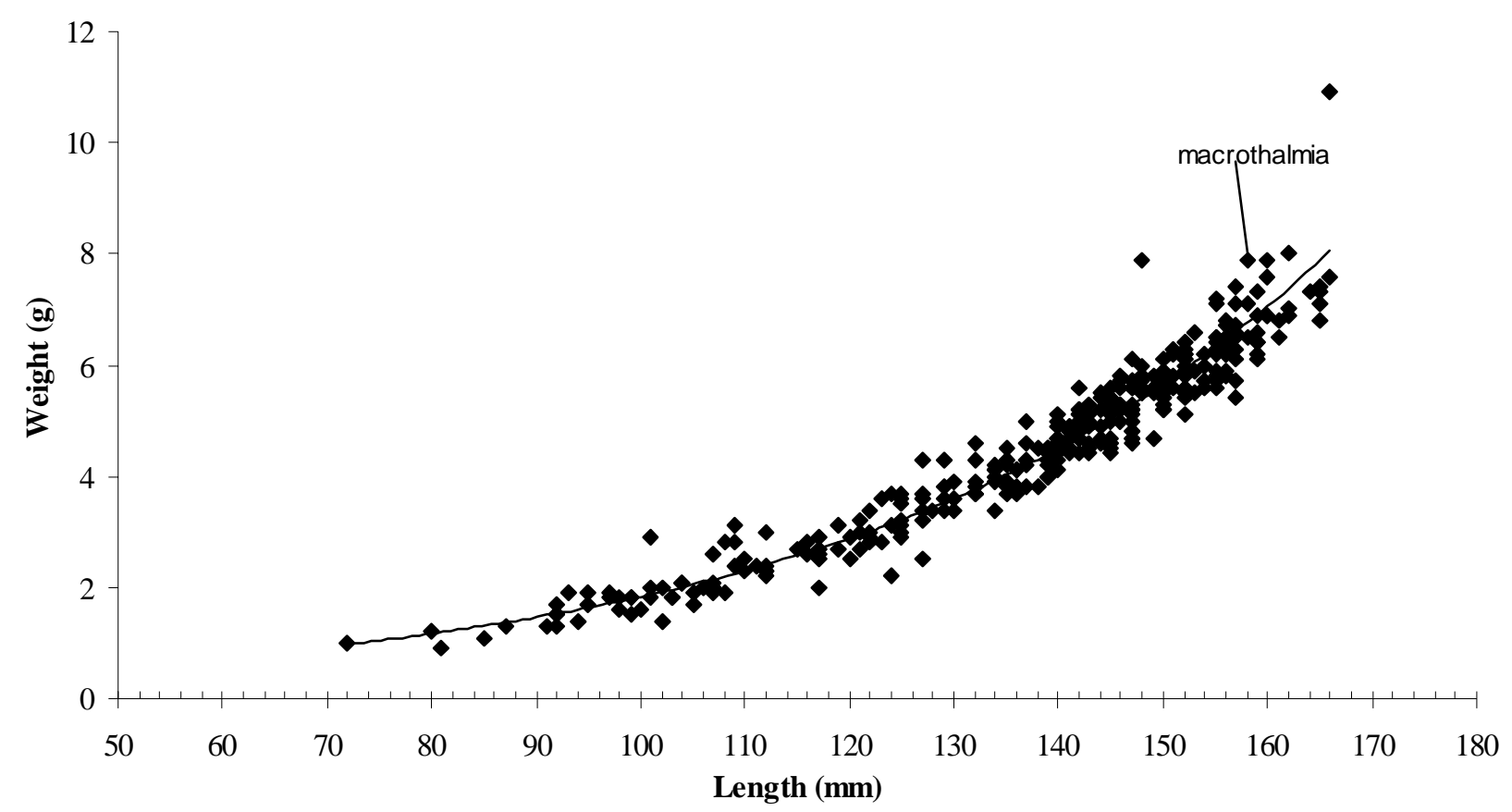

Figure 9. Pacific lamprey ammocoete and macrothalmia (electroshocked) length and weight relationship, (N=333) Red River, ID, 2000-01.

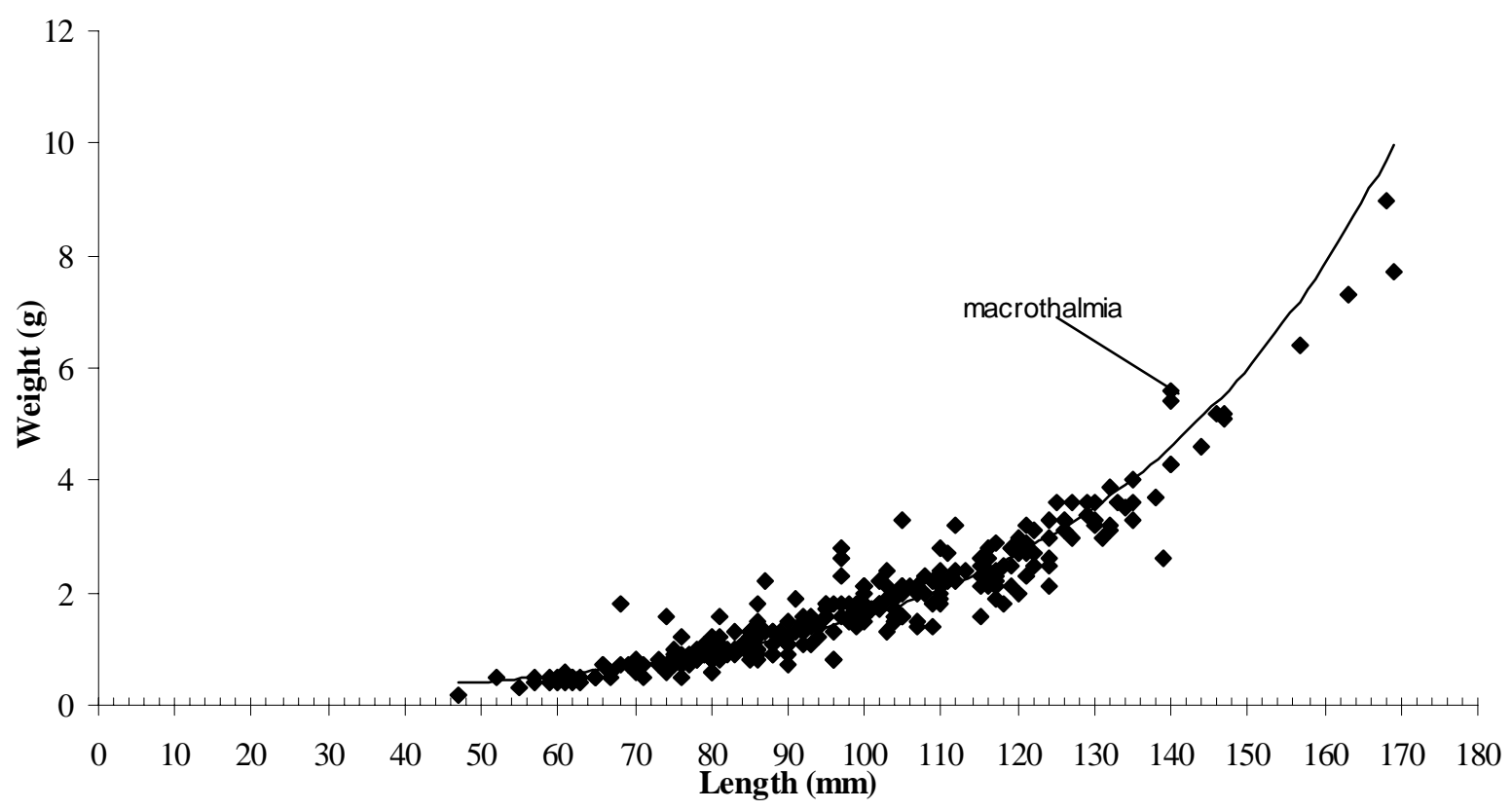

Figure 10. Pacific lamprey ammocoete and macrothalmia (electroshocked) length and weight relationship, (N=301) South Fork Clearwater River, ID, 2000-01. 


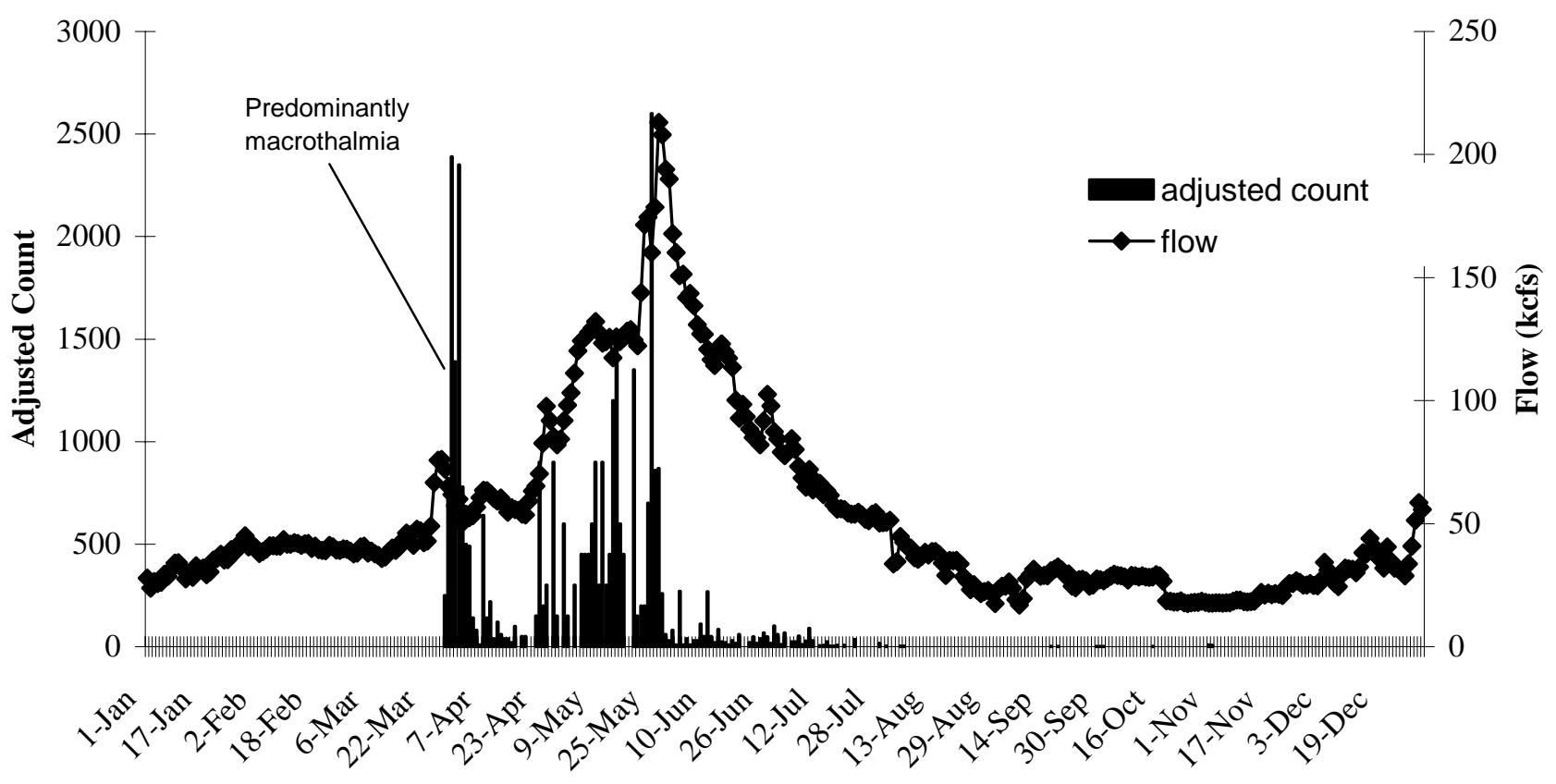

Figure 11. Pacific lamprey ammocoete and macrothalmia adjusted bypass/collection count and Snake River flows, Lower Granite Dam, WA, 1998. 


\section{DISCUSSION}

It is not known if Pacific lamprey densities observed in 2000-02 reflect overall low population numbers in the S.F. Clearwater River drainage. The number of spawning adults in the S.F. Clearwater River basin is suspected to have totaled fewer than 50 Pacific lamprey annually 1998-2001. The S.F. Clearwater River estimated spawning adult escapement is based on Lower Granite dam passage of less than 320 adults in the 1998-2001 period and assumption of even distribution of Pacific lamprey adults into the Grande Ronde, Imnaha, Salmon, and Clearwater Rivers with one-half of Clearwater River drainage spawners returning to the S.F. Clearwater River.

Presence-absence sampling in 2000-2002 in the S.F. Clearwater River basin indicates distribution of Pacific lamprey in the basin is restricted to the mainstem S.F. Clearwater River and the lower Red River. Suitable habitat remains in the upper Red River, Newsome Creek, American River, and Crooked River, but Pacific lamprey ammocoetes and macrothalmia were not found. Pacific lamprey ammocoete distribution in the Selway River and Lochsa River drainages was limited to mainstem reaches. Of the 70 sites sampled in the Clearwater River basin in 2002, only 15 produced Pacific lamprey. Population numbers are minimal and distribution restricted to the remaining preferred habitat in the entire Clearwater River basin. 


\section{LITERATURE CITED}

Beamish, R. J., and C. D. Levings. 1991. Abundance and Freshwater Migrations of the Anadromous Parasitic Lamprey, Lampetra tridentata, in a Tributary of the Fraser River, British Columbia. Canadian Journal of Fisheries and Aquatic Sciences 48:1250-1263.

Beamish, F. W., and T. E. Medland. 1988. Age Determination for Lampreys. Transactions of the American Fisheries Society 117:63-71.

Galbreath, J. 1979. Columbia River Colossus, the White Sturgeon. Oregon Wildlife March 1979:3-8.

Merrell, T. R. 1959. Gull Food Habits on the Columbia River. Research Briefs, Fish Commission of Oregon 7(1):82.

Scott, W. B., and E. J. Crossman. 1973. Freshwater Fishes of Canada. Fisheries Research Board of Canada Bulletin 184:42-45.

USDA Forest Service. 1998. South Fork Clearwater River Landscape Assessment. USDA Forest Service. Nez Perce National Forest. 1998:I:210.

White, C. R. 1954. The Age, Growth, and Migration of Steelhead Trout in the Clearwater River, ID. University of Idaho, M. S. Thesis.

1954:65. 
APPENDIX A 
Table A1. Pacific lamprey length, weight, and mark information from Red River, ID, 2002.

\begin{tabular}{|c|c|c|c|c|c|c|c|c|c|c|}
\hline $\begin{array}{c}\text { Date } \\
\text { Electrofished }\end{array}$ & $\begin{array}{c}\mathrm{Km} / \\
\text { Reach }\end{array}$ & $\begin{array}{c}\text { Habitat } \\
\text { Type }\end{array}$ & $\begin{array}{c}\text { Area } \\
\text { Fished } \\
\left(\mathrm{m}^{2}\right)\end{array}$ & $\begin{array}{c}\text { Time } \\
\text { Fished } \\
\text { (Min.) }\end{array}$ & $\begin{array}{l}\text { Lamprey } \\
\text { Captured }\end{array}$ & $\begin{array}{c}\text { Lamprey } \\
\text { Measured }\end{array}$ & $\begin{array}{l}\text { Length } \\
(\mathrm{mm})\end{array}$ & $\begin{array}{l}\text { Weight } \\
(\mathrm{g})\end{array}$ & Mark $^{1}$ & Phase $^{2}$ \\
\hline $8 / 10 / 2002$ & $06 / 03$ & RIP & 288.62 & 224 & 43 & 43 & 126 & 2.6 & NO MARK & $\mathrm{U}$ \\
\hline $8 / 10 / 2002$ & $06 / 03$ & RIP & & & & & 114 & 2.5 & NO MARK & $\mathrm{U}$ \\
\hline $8 / 10 / 2002$ & $06 / 03$ & RIP & & & & & 150 & 5.6 & NO MARK & $\mathrm{U}$ \\
\hline $8 / 10 / 2002$ & $06 / 03$ & RIP & & & & & 137 & 3.9 & NO MARK & $\mathrm{U}$ \\
\hline $8 / 10 / 2002$ & $06 / 03$ & RIP & & & & & 149 & 5.2 & NO MARK & $\mathrm{U}$ \\
\hline $8 / 10 / 2002$ & $06 / 03$ & RIP & & & & & 152 & 5.1 & NO MARK & $\mathrm{U}$ \\
\hline $8 / 10 / 2002$ & $06 / 03$ & RIP & & & & & 130 & 3.6 & NO MARK & $\mathrm{U}$ \\
\hline $8 / 10 / 2002$ & $06 / 03$ & RIP & & & & & 115 & 2.2 & NO MARK & $\mathrm{U}$ \\
\hline $8 / 10 / 2002$ & $06 / 03$ & RIP & & & & & 105 & 1.4 & NO MARK & $\mathrm{U}$ \\
\hline $8 / 10 / 2002$ & $06 / 03$ & RIP & & & & & 146 & 5.0 & NO MARK & $\mathrm{U}$ \\
\hline $8 / 10 / 2002$ & $06 / 03$ & RIP & & & & & 126 & 3.0 & NO MARK & $\mathrm{U}$ \\
\hline $8 / 10 / 2002$ & $06 / 03$ & RIP & & & & & 140 & 4.1 & NO MARK & $\mathrm{U}$ \\
\hline $8 / 10 / 2002$ & $06 / 03$ & RIP & & & & & 116 & 2.0 & NO MARK & $\mathrm{U}$ \\
\hline $8 / 10 / 2002$ & $06 / 03$ & RIP & & & & & 145 & 4.7 & NO MARK & $\mathrm{U}$ \\
\hline $8 / 10 / 2002$ & $06 / 03$ & RIP & & & & & 131 & 3.7 & NO MARK & $\mathrm{U}$ \\
\hline $8 / 10 / 2002$ & $06 / 03$ & RIP & & & & & 123 & 2.7 & NO MARK & $\mathrm{U}$ \\
\hline $8 / 10 / 2002$ & $06 / 03$ & RIP & & & & & 100 & 1.6 & NO MARK & $\mathrm{U}$ \\
\hline $8 / 10 / 2002$ & $06 / 03$ & RIP & & & & & 140 & 4.5 & NO MARK & $\mathrm{U}$ \\
\hline $8 / 10 / 2002$ & $06 / 03$ & RIP & & & & & 132 & 3.8 & NO MARK & $\mathrm{U}$ \\
\hline $8 / 10 / 2002$ & $06 / 03$ & RIP & & & & & 119 & 2.3 & NO MARK & $\mathrm{U}$ \\
\hline $8 / 10 / 2002$ & $06 / 03$ & RIP & & & & & 104 & 1.8 & NO MARK & $\mathrm{U}$ \\
\hline $8 / 10 / 2002$ & $06 / 03$ & RIP & & & & & 95 & 1.4 & NO MARK & $\mathrm{U}$ \\
\hline $8 / 10 / 2002$ & $06 / 03$ & RIP & & & & & 116 & 2.2 & NO MARK & $\mathrm{U}$ \\
\hline $8 / 10 / 2002$ & $06 / 03$ & RIP & & & & & 124 & 3.0 & NO MARK & $\mathrm{U}$ \\
\hline $8 / 10 / 2002$ & $06 / 03$ & RIP & & & & & 134 & 3.4 & NO MARK & $\mathrm{U}$ \\
\hline $8 / 10 / 2002$ & $06 / 03$ & RIP & & & & & 134 & 3.3 & NO MARK & $\mathrm{U}$ \\
\hline $8 / 10 / 2002$ & $06 / 03$ & RIP & & & & & 129 & 3.2 & NO MARK & $\mathrm{U}$ \\
\hline $8 / 10 / 2002$ & $06 / 03$ & RIP & & & & & 105 & 1.9 & NO MARK & $\mathrm{U}$ \\
\hline $8 / 10 / 2002$ & $06 / 03$ & RIP & & & & & 124 & 2.8 & NO MARK & $\mathrm{U}$ \\
\hline $8 / 10 / 2002$ & $06 / 03$ & RIP & & & & & 111 & 2.2 & NO MARK & $\mathrm{U}$ \\
\hline $8 / 10 / 2002$ & $06 / 03$ & RIP & & & & & 136 & 3.2 & NO MARK & $\mathrm{U}$ \\
\hline $8 / 10 / 2002$ & $06 / 03$ & RIP & & & & & 104 & 1.8 & NO MARK & $\mathrm{U}$ \\
\hline $8 / 10 / 2002$ & $06 / 03$ & RIP & & & & & 130 & 3.3 & NO MARK & $\mathrm{U}$ \\
\hline $8 / 10 / 2002$ & $06 / 03$ & RIP & & & & & 150 & 5.4 & NO MARK & $\mathrm{U}$ \\
\hline $8 / 10 / 2002$ & $06 / 03$ & RIP & & & & & 125 & 2.9 & NO MARK & $\mathrm{U}$ \\
\hline $8 / 10 / 2002$ & $06 / 03$ & RIP & & & & & 123 & 2.9 & NO MARK & $\mathrm{U}$ \\
\hline $8 / 10 / 2002$ & $06 / 03$ & RIP & & & & & 111 & 2.3 & NO MARK & $\mathrm{U}$ \\
\hline $8 / 10 / 2002$ & $06 / 03$ & RIP & & & & & 105 & 1.9 & NO MARK & $\mathrm{U}$ \\
\hline $8 / 10 / 2002$ & $06 / 03$ & RIP & & & & & 117 & 2.8 & NO MARK & $\mathrm{U}$ \\
\hline $8 / 10 / 2002$ & $06 / 03$ & RIP & & & & & 126 & 3.2 & NO MARK & $\mathrm{U}$ \\
\hline $8 / 10 / 2002$ & $06 / 03$ & RIP & & & & & 136 & 4.0 & NO MARK & $\mathrm{U}$ \\
\hline $8 / 10 / 2002$ & $06 / 03$ & RIP & & & & & 130 & 3.6 & NO MARK & $\mathrm{U}$ \\
\hline $8 / 10 / 2002$ & $06 / 03$ & RIP & & & & & 134 & 3.8 & NO MARK & $\mathrm{U}$ \\
\hline $8 / 11 / 2002$ & $06 / 03$ & LSP & 110.95 & 162 & 169 & 168 & 153 & 5.7 & NO MARK & $\mathrm{U}$ \\
\hline $8 / 11 / 2002$ & $06 / 03$ & LSP & & & & & 120 & 2.6 & NO MARK & $\mathrm{U}$ \\
\hline
\end{tabular}

1. RCO-Right center orange

2. T-transformed; U-transformed 
Table Al. (con't).

\begin{tabular}{|c|c|c|c|c|c|c|c|c|c|c|}
\hline $\begin{array}{c}\text { Date } \\
\text { Electrofished }\end{array}$ & $\begin{array}{l}\mathrm{Km} / \\
\text { Reach }\end{array}$ & $\begin{array}{l}\text { Habitat } \\
\text { Type }\end{array}$ & $\begin{array}{c}\text { Area } \\
\text { Fished } \\
\left(\mathrm{m}^{2}\right)\end{array}$ & $\begin{array}{l}\text { Time } \\
\text { Fished } \\
\text { (Min.) }\end{array}$ & $\begin{array}{l}\text { Lamprey } \\
\text { Captured }\end{array}$ & $\begin{array}{l}\text { Lamprey } \\
\text { Measured }\end{array}$ & $\begin{array}{l}\text { Length } \\
(\mathrm{mm})\end{array}$ & $\begin{array}{l}\text { Weight } \\
(\mathrm{g})\end{array}$ & Mark $^{1}$ & Phase $^{2}$ \\
\hline $8 / 11 / 2002$ & $06 / 03$ & LSP & & & & & 110 & 2.2 & NO MARK & $\mathrm{U}$ \\
\hline $8 / 11 / 2002$ & $06 / 03$ & LSP & & & & & 129 & 3.0 & NO MARK & $\mathrm{U}$ \\
\hline $8 / 11 / 2002$ & $06 / 03$ & LSP & & & & & 111 & 2.7 & NO MARK & $\mathrm{U}$ \\
\hline $8 / 11 / 2002$ & $06 / 03$ & LSP & & & & & 134 & 3.7 & NO MARK & $\mathrm{U}$ \\
\hline $8 / 11 / 2002$ & $06 / 03$ & LSP & & & & & 116 & 2.6 & NO MARK & $\mathrm{U}$ \\
\hline $8 / 11 / 2002$ & $06 / 03$ & LSP & & & & & 111 & 2.2 & NO MARK & $\mathrm{U}$ \\
\hline $8 / 11 / 2002$ & $06 / 03$ & LSP & & & & & 125 & 3.1 & NO MARK & $\mathrm{U}$ \\
\hline $8 / 11 / 2002$ & $06 / 03$ & LSP & & & & & 114 & 2.1 & NO MARK & $\mathrm{U}$ \\
\hline $8 / 11 / 2002$ & $06 / 03$ & LSP & & & & & 127 & 3.4 & NO MARK & $\mathrm{U}$ \\
\hline $8 / 11 / 2002$ & $06 / 03$ & LSP & & & & & 112 & 1.6 & NO MARK & $\mathrm{U}$ \\
\hline $8 / 11 / 2002$ & $06 / 03$ & LSP & & & & & 120 & 2.7 & NO MARK & $\mathrm{U}$ \\
\hline $8 / 11 / 2002$ & $06 / 03$ & LSP & & & & & 134 & 3.8 & NO MARK & $\mathrm{U}$ \\
\hline $8 / 11 / 2002$ & $06 / 03$ & LSP & & & & & 137 & 4.1 & NO MARK & $\mathrm{U}$ \\
\hline $8 / 11 / 2002$ & $06 / 03$ & LSP & & & & & 126 & na & NO MARK & $\mathrm{U}$ \\
\hline $8 / 11 / 2002$ & $06 / 03$ & LSP & & & & & 147 & 4.6 & NO MARK & $\mathrm{U}$ \\
\hline $8 / 11 / 2002$ & $06 / 03$ & LSP & & & & & 136 & 4.5 & NO MARK & $\mathrm{U}$ \\
\hline $8 / 11 / 2002$ & $06 / 03$ & LSP & & & & & 135 & 3.8 & NO MARK & $\mathrm{U}$ \\
\hline $8 / 11 / 2002$ & $06 / 03$ & LSP & & & & & 121 & 2.7 & NO MARK & $\mathrm{U}$ \\
\hline $8 / 11 / 2002$ & $06 / 03$ & LSP & & & & & 104 & 1.7 & NO MARK & $\mathrm{U}$ \\
\hline $8 / 11 / 2002$ & $06 / 03$ & LSP & & & & & 108 & 1.9 & NO MARK & $\mathrm{U}$ \\
\hline $8 / 11 / 2002$ & $06 / 03$ & LSP & & & & & 114 & 2.1 & NO MARK & $\mathrm{U}$ \\
\hline $8 / 11 / 2002$ & $06 / 03$ & LSP & & & & & 99 & 1.9 & NO MARK & $\mathrm{U}$ \\
\hline $8 / 11 / 2002$ & $06 / 03$ & LSP & & & & & 110 & 2.4 & NO MARK & $\mathrm{U}$ \\
\hline $8 / 11 / 2002$ & $06 / 03$ & LSP & & & & & 106 & 2.2 & NO MARK & $\mathrm{U}$ \\
\hline $8 / 11 / 2002$ & $06 / 03$ & LSP & & & & & 128 & 3.0 & NO MARK & $\mathrm{U}$ \\
\hline $8 / 11 / 2002$ & $06 / 03$ & LSP & & & & & 141 & 4.6 & NO MARK & $\mathrm{U}$ \\
\hline $8 / 11 / 2002$ & $06 / 03$ & LSP & & & & & 149 & 4.8 & NO MARK & $\mathrm{U}$ \\
\hline $8 / 11 / 2002$ & $06 / 03$ & LSP & & & & & 139 & 4.3 & NO MARK & $\mathrm{U}$ \\
\hline $8 / 11 / 2002$ & $06 / 03$ & LSP & & & & & 146 & 4.4 & NO MARK & $\mathrm{U}$ \\
\hline $8 / 11 / 2002$ & $06 / 03$ & LSP & & & & & 142 & 4.6 & NO MARK & $\mathrm{U}$ \\
\hline $8 / 11 / 2002$ & $06 / 03$ & LSP & & & & & 109 & 2.0 & NO MARK & $\mathrm{U}$ \\
\hline $8 / 11 / 2002$ & $06 / 03$ & LSP & & & & & 104 & 1.9 & NO MARK & $\mathrm{U}$ \\
\hline $8 / 11 / 2002$ & $06 / 03$ & LSP & & & & & 121 & 2.9 & NO MARK & $\mathrm{U}$ \\
\hline $8 / 11 / 2002$ & $06 / 03$ & LSP & & & & & 109 & 2.0 & NO MARK & $\mathrm{U}$ \\
\hline $8 / 11 / 2002$ & $06 / 03$ & LSP & & & & & 138 & 4.5 & NO MARK & $\mathrm{U}$ \\
\hline $8 / 11 / 2002$ & $06 / 03$ & LSP & & & & & 90 & 1.4 & NO MARK & $\mathrm{U}$ \\
\hline $8 / 11 / 2002$ & $06 / 03$ & LSP & & & & & 113 & 2.3 & NO MARK & $\mathrm{U}$ \\
\hline $8 / 11 / 2002$ & $06 / 03$ & LSP & & & & & 106 & 1.7 & NO MARK & $\mathrm{U}$ \\
\hline $8 / 11 / 2002$ & $06 / 03$ & LSP & & & & & 145 & 4.6 & NO MARK & $\mathrm{U}$ \\
\hline $8 / 11 / 2002$ & $06 / 03$ & LSP & & & & & 143 & 4.7 & NO MARK & $\mathrm{U}$ \\
\hline $8 / 11 / 2002$ & $06 / 03$ & LSP & & & & & 145 & 5.0 & NO MARK & $\mathrm{U}$ \\
\hline $8 / 11 / 2002$ & $06 / 03$ & LSP & & & & & 129 & 3.3 & NO MARK & $\mathrm{U}$ \\
\hline $8 / 11 / 2002$ & $06 / 03$ & LSP & & & & & 108 & 1.8 & NO MARK & $\mathrm{U}$ \\
\hline $8 / 11 / 2002$ & $06 / 03$ & LSP & & & & & 103 & 1.6 & NO MARK & $\mathrm{U}$ \\
\hline $8 / 11 / 2002$ & $06 / 03$ & LSP & & & & & 148 & 5.0 & NO MARK & $\mathrm{U}$ \\
\hline
\end{tabular}

1 RCO-Right center orange

2 T-transformed; U-transformed 
Table A1.(con't.)

\begin{tabular}{|c|c|c|c|c|c|c|c|c|c|c|}
\hline $\begin{array}{c}\text { Date } \\
\text { Electrofished }\end{array}$ & $\begin{array}{l}\mathrm{Km} / \\
\text { Reach }\end{array}$ & $\begin{array}{c}\text { Habitat } \\
\text { Type }\end{array}$ & $\begin{array}{c}\text { Area } \\
\text { Fished } \\
\left(\mathrm{m}^{2}\right)\end{array}$ & $\begin{array}{c}\text { Time } \\
\text { Fished } \\
\text { (Min.) }\end{array}$ & $\begin{array}{l}\text { Lamprey } \\
\text { Captured }\end{array}$ & $\begin{array}{l}\text { Lamprey } \\
\text { Measured }\end{array}$ & $\begin{array}{l}\text { Length } \\
(\mathrm{mm})\end{array}$ & $\begin{array}{l}\text { Weight } \\
\text { (g) }\end{array}$ & Mark $^{1}$ & Phase $^{2}$ \\
\hline $8 / 11 / 2002$ & $06 / 03$ & LSP & & & & & 109 & 2.0 & NO MARK & $\mathrm{U}$ \\
\hline $8 / 11 / 2002$ & $06 / 03$ & LSP & & & & & 120 & 3.0 & NO MARK & $\mathrm{U}$ \\
\hline $8 / 11 / 2002$ & $06 / 03$ & LSP & & & & & 112 & 2.3 & NO MARK & $\mathrm{U}$ \\
\hline $8 / 11 / 2002$ & $06 / 03$ & LSP & & & & & 117 & 2.6 & NO MARK & $\mathrm{U}$ \\
\hline $8 / 11 / 2002$ & $06 / 03$ & LSP & & & & & 111 & 2.1 & NO MARK & $\mathrm{U}$ \\
\hline $8 / 11 / 2002$ & $06 / 03$ & LSP & & & & & 114 & 2.5 & NO MARK & $\mathrm{U}$ \\
\hline $8 / 11 / 2002$ & $06 / 03$ & LSP & & & & & 106 & 2.5 & NO MARK & $\mathrm{U}$ \\
\hline $8 / 11 / 2002$ & $06 / 03$ & LSP & & & & & 107 & 1.8 & NO MARK & $\mathrm{U}$ \\
\hline $8 / 11 / 2002$ & $06 / 03$ & LSP & & & & & 104 & 1.8 & NO MARK & $\mathrm{U}$ \\
\hline $8 / 11 / 2002$ & $06 / 03$ & LSP & & & & & 96 & 1.6 & NO MARK & $\mathrm{U}$ \\
\hline $8 / 11 / 2002$ & $06 / 03$ & LSP & & & & & 111 & 2.1 & NO MARK & $\mathrm{U}$ \\
\hline $8 / 11 / 2002$ & $06 / 03$ & LSP & & & & & 107 & 1.7 & NO MARK & $\mathrm{U}$ \\
\hline $8 / 11 / 2002$ & $06 / 03$ & LSP & & & & & 121 & 2.7 & NO MARK & $\mathrm{U}$ \\
\hline $8 / 11 / 2002$ & $06 / 03$ & LSP & & & & & 140 & 5.1 & NO MARK & $\mathrm{U}$ \\
\hline $8 / 11 / 2002$ & $06 / 03$ & LSP & & & & & 105 & 2.0 & NO MARK & $\mathrm{U}$ \\
\hline $8 / 11 / 2002$ & $06 / 03$ & LSP & & & & & 143 & 4.2 & NO MARK & $\mathrm{U}$ \\
\hline $8 / 11 / 2002$ & $06 / 03$ & LSP & & & & & 103 & 1.9 & NO MARK & $\mathrm{U}$ \\
\hline $8 / 11 / 2002$ & $06 / 03$ & LSP & & & & & 118 & 2.8 & NO MARK & $\mathrm{U}$ \\
\hline $8 / 11 / 2002$ & $06 / 03$ & LSP & & & & & 125 & 3.2 & NO MARK & $\mathrm{U}$ \\
\hline $8 / 11 / 2002$ & 06/03 & LSP & & & & & 137 & 4.6 & NO MARK & $\mathrm{U}$ \\
\hline $8 / 11 / 2002$ & $06 / 03$ & LSP & & & & & 110 & 2.5 & NO MARK & $\mathrm{U}$ \\
\hline $8 / 11 / 2002$ & $06 / 03$ & LSP & & & & & 112 & 2.6 & NO MARK & $\mathrm{U}$ \\
\hline $8 / 11 / 2002$ & $06 / 03$ & LSP & & & & & 117 & 2.8 & NO MARK & $\mathrm{U}$ \\
\hline $8 / 11 / 2002$ & $06 / 03$ & LSP & & & & & 141 & 4.2 & NO MARK & $\mathrm{U}$ \\
\hline $8 / 11 / 2002$ & $06 / 03$ & LSP & & & & & 119 & 3.3 & NO MARK & $\mathrm{U}$ \\
\hline $8 / 11 / 2002$ & $06 / 03$ & LSP & & & & & 102 & 1.7 & NO MARK & $\mathrm{U}$ \\
\hline $8 / 11 / 2002$ & $06 / 03$ & LSP & & & & & 105 & 2.1 & NO MARK & $\mathrm{U}$ \\
\hline $8 / 11 / 2002$ & $06 / 03$ & LSP & & & & & 131 & 3.7 & NO MARK & $\mathrm{U}$ \\
\hline $8 / 11 / 2002$ & $06 / 03$ & LSP & & & & & 113 & 2.1 & NO MARK & $\mathrm{U}$ \\
\hline $8 / 11 / 2002$ & $06 / 03$ & LSP & & & & & 126 & 3.6 & NO MARK & $\mathrm{U}$ \\
\hline $8 / 11 / 2002$ & $06 / 03$ & LSP & & & & & 139 & 3.6 & NO MARK & $\mathrm{U}$ \\
\hline $8 / 11 / 2002$ & $06 / 03$ & LSP & & & & & 130 & -- & NO MARK & $\mathrm{U}$ \\
\hline $8 / 11 / 2002$ & $06 / 03$ & LSP & & & & & 131 & 3.9 & NO MARK & $\mathrm{U}$ \\
\hline $8 / 11 / 2002$ & $06 / 03$ & LSP & & & & & 111 & 2.1 & NO MARK & $\mathrm{U}$ \\
\hline $8 / 11 / 2002$ & $06 / 03$ & LSP & & & & & 129 & 3.4 & NO MARK & $\mathrm{U}$ \\
\hline $8 / 11 / 2002$ & $06 / 03$ & LSP & & & & & 120 & 2.9 & NO MARK & $\mathrm{U}$ \\
\hline $8 / 11 / 2002$ & $06 / 03$ & LSP & & & & & 108 & 1.8 & NO MARK & $\mathrm{U}$ \\
\hline $8 / 11 / 2002$ & $06 / 03$ & LSP & & & & & 122 & 2.4 & NO MARK & $\mathrm{U}$ \\
\hline $8 / 11 / 2002$ & $06 / 03$ & LSP & & & & & 97 & 1.6 & NO MARK & $\mathrm{U}$ \\
\hline $8 / 11 / 2002$ & $06 / 03$ & LSP & & & & & 124 & 3.2 & NO MARK & $\mathrm{U}$ \\
\hline $8 / 11 / 2002$ & $06 / 03$ & LSP & & & & & 119 & 2.7 & NO MARK & $\mathrm{U}$ \\
\hline $8 / 11 / 2002$ & $06 / 03$ & LSP & & & & & 129 & 3.3 & NO MARK & $\mathrm{U}$ \\
\hline $8 / 11 / 2002$ & $06 / 03$ & LSP & & & & & 102 & 1.6 & NO MARK & $\mathrm{U}$ \\
\hline $8 / 11 / 2002$ & $06 / 03$ & LSP & & & & & 105 & 2.1 & NO MARK & $\mathrm{U}$ \\
\hline $8 / 11 / 2002$ & $06 / 03$ & LSP & & & & & 98 & 1.5 & NO MARK & $\mathrm{U}$ \\
\hline
\end{tabular}

1 RCO-Right center orange

2 T-transformed; U-transformed 
Table A1. (con't).

\begin{tabular}{|c|c|c|c|c|c|c|c|c|c|c|}
\hline $\begin{array}{c}\text { Date } \\
\text { Electrofished }\end{array}$ & $\begin{array}{c}\mathrm{Km} / \\
\text { Reach }\end{array}$ & $\begin{array}{c}\text { Habitat } \\
\text { Type }\end{array}$ & $\begin{array}{c}\text { Area } \\
\text { Fished } \\
\left(\mathrm{m}^{2}\right) \\
\end{array}$ & $\begin{array}{c}\text { Time } \\
\text { Fished } \\
\text { (Min.) }\end{array}$ & $\begin{array}{l}\text { Lamprey } \\
\text { Captured }\end{array}$ & $\begin{array}{c}\text { Lamprey } \\
\text { Measured }\end{array}$ & $\begin{array}{l}\text { Length } \\
(\mathrm{mm})\end{array}$ & $\begin{array}{c}\text { Weight } \\
(\mathrm{g})\end{array}$ & Mark $^{1}$ & Phase $^{2}$ \\
\hline $8 / 11 / 2002$ & $06 / 03$ & LSP & & & & & 111 & 2.4 & NO MARK & $\mathrm{U}$ \\
\hline $8 / 11 / 2002$ & $06 / 03$ & LSP & & & & & 115 & 2.5 & NO MARK & $\mathrm{U}$ \\
\hline $8 / 11 / 2002$ & $06 / 03$ & LSP & & & & & 106 & 1.9 & NO MARK & $\mathrm{U}$ \\
\hline $8 / 11 / 2002$ & $06 / 03$ & LSP & & & & & 99 & 1.5 & NO MARK & $\mathrm{U}$ \\
\hline $8 / 11 / 2002$ & $06 / 03$ & LSP & & & & & 108 & 2.0 & NO MARK & $\mathrm{U}$ \\
\hline $8 / 11 / 2002$ & $06 / 03$ & LSP & & & & & 94 & 1.7 & NO MARK & $\mathrm{U}$ \\
\hline $8 / 11 / 2002$ & $06 / 03$ & LSP & & & & & 107 & 2.2 & NO MARK & $\mathrm{U}$ \\
\hline $8 / 11 / 2002$ & $06 / 03$ & LSP & & & & & 104 & 1.9 & NO MARK & $\mathrm{U}$ \\
\hline $8 / 11 / 2002$ & $06 / 03$ & LSP & & & & & 99 & 1.8 & NO MARK & $\mathrm{U}$ \\
\hline $8 / 11 / 2002$ & $06 / 03$ & LSP & & & & & 113 & 2.7 & NO MARK & $\mathrm{U}$ \\
\hline $8 / 11 / 2002$ & $06 / 03$ & LSP & & & & & 97 & 2.0 & NO MARK & $\mathrm{U}$ \\
\hline $8 / 11 / 2002$ & $06 / 03$ & LSP & & & & & 142 & 5.2 & NO MARK & $\mathrm{U}$ \\
\hline $8 / 11 / 2002$ & $06 / 03$ & LSP & & & & & 128 & 3.9 & NO MARK & $\mathrm{U}$ \\
\hline $8 / 11 / 2002$ & $06 / 03$ & LSP & & & & & 107 & 2.0 & NO MARK & $\mathrm{U}$ \\
\hline $8 / 11 / 2002$ & $06 / 03$ & LSP & & & & & 134 & 4.1 & NO MARK & $\mathrm{U}$ \\
\hline $8 / 11 / 2002$ & $06 / 03$ & LSP & & & & & 105 & 2.1 & NO MARK & $\mathrm{U}$ \\
\hline $8 / 11 / 2002$ & $06 / 03$ & LSP & & & & & 148 & 5.6 & NO MARK & $\mathrm{U}$ \\
\hline $8 / 11 / 2002$ & $06 / 03$ & LSP & & & & & 108 & 2.2 & NO MARK & U \\
\hline $8 / 11 / 2002$ & $06 / 03$ & LSP & & & & & 128 & 3.3 & NO MARK & U \\
\hline $8 / 11 / 2002$ & $06 / 03$ & LSP & & & & & 144 & 4.6 & NO MARK & $\mathrm{U}$ \\
\hline $8 / 11 / 2002$ & $06 / 03$ & LSP & & & & & 130 & 3.7 & NO MARK & $\mathrm{U}$ \\
\hline $8 / 11 / 2002$ & $06 / 03$ & LSP & & & & & 131 & 3.9 & NO MARK & $\mathrm{U}$ \\
\hline $8 / 11 / 2002$ & $06 / 03$ & LSP & & & & & 123 & 3.1 & NO MARK & $\mathrm{U}$ \\
\hline $8 / 11 / 2002$ & $06 / 03$ & LSP & & & & & 109 & 2.0 & NO MARK & $\mathrm{U}$ \\
\hline $8 / 11 / 2002$ & $06 / 03$ & LSP & & & & & 136 & 4.1 & NO MARK & $\mathrm{U}$ \\
\hline $8 / 11 / 2002$ & $06 / 03$ & LSP & & & & & 100 & 1.7 & NO MARK & $\mathrm{U}$ \\
\hline $8 / 11 / 2002$ & $06 / 03$ & LSP & & & & & 106 & 2.1 & NO MARK & $\mathrm{U}$ \\
\hline $8 / 11 / 2002$ & $06 / 03$ & LSP & & & & & 110 & 2.4 & NO MARK & $\mathrm{U}$ \\
\hline $8 / 11 / 2002$ & $06 / 03$ & LSP & & & & & 94 & 1.4 & NO MARK & $\mathrm{U}$ \\
\hline $8 / 11 / 2002$ & $06 / 03$ & LSP & & & & & 96 & 1.8 & NO MARK & $\mathrm{U}$ \\
\hline $8 / 11 / 2002$ & $06 / 03$ & LSP & & & & & 100 & 1.8 & NO MARK & $\mathrm{U}$ \\
\hline $8 / 11 / 2002$ & $06 / 03$ & LSP & & & & & 119 & 2.8 & NO MARK & $\mathrm{U}$ \\
\hline $8 / 11 / 2002$ & $06 / 03$ & LSP & & & & & 100 & 1.8 & NO MARK & U \\
\hline $8 / 11 / 2002$ & $06 / 03$ & LSP & & & & & 134 & 4.1 & NO MARK & $\mathrm{U}$ \\
\hline $8 / 11 / 2002$ & $06 / 03$ & LSP & & & & & 132 & 4.0 & NO MARK & $\mathrm{U}$ \\
\hline $8 / 11 / 2002$ & $06 / 03$ & LSP & & & & & 150 & 5.4 & NO MARK & $\mathrm{U}$ \\
\hline $8 / 11 / 2002$ & $06 / 03$ & LSP & & & & & 111 & 2.3 & NO MARK & $\mathrm{U}$ \\
\hline $8 / 11 / 2002$ & $06 / 03$ & LSP & & & & & 103 & 1.8 & NO MARK & $\mathrm{U}$ \\
\hline $8 / 11 / 2002$ & $06 / 03$ & LSP & & & & & 97 & 1.6 & NO MARK & $\mathrm{U}$ \\
\hline $8 / 11 / 2002$ & $06 / 03$ & LSP & & & & & 124 & 3.2 & NO MARK & $\mathrm{U}$ \\
\hline $8 / 11 / 2002$ & $06 / 03$ & LSP & & & & & 97 & 1.5 & NO MARK & $\mathrm{U}$ \\
\hline $8 / 11 / 2002$ & $06 / 03$ & LSP & & & & & 113 & 2.2 & NO MARK & $\mathrm{U}$ \\
\hline $8 / 11 / 2002$ & $06 / 03$ & LSP & & & & & 103 & 2.0 & NO MARK & $\mathrm{U}$ \\
\hline $8 / 11 / 2002$ & $06 / 03$ & LSP & & & & & 107 & 2.2 & NO MARK & $\mathrm{U}$ \\
\hline $8 / 11 / 2002$ & $06 / 03$ & LSP & & & & & 105 & 2.2 & NO MARK & $\mathrm{U}$ \\
\hline
\end{tabular}

1 RCO-Right center orange

2 T-transformed; U-untransformed 
Table A1. (con't).

\begin{tabular}{|c|c|c|c|c|c|c|c|c|c|c|}
\hline $\begin{array}{c}\text { Date } \\
\text { Electrofished }\end{array}$ & $\begin{array}{l}\mathrm{Km} / \\
\text { Reach }\end{array}$ & $\begin{array}{c}\text { Habitat } \\
\text { Type }\end{array}$ & $\begin{array}{c}\text { Area } \\
\text { Fished } \\
\left(\mathrm{m}^{2}\right)\end{array}$ & $\begin{array}{l}\text { Time } \\
\text { Fished } \\
\text { (Min.) }\end{array}$ & $\begin{array}{l}\text { Lamprey } \\
\text { Captured }\end{array}$ & $\begin{array}{c}\text { Lamprey } \\
\text { Measured }\end{array}$ & $\begin{array}{c}\text { Length } \\
(\mathrm{mm})\end{array}$ & $\begin{array}{c}\text { Weight } \\
(\mathrm{g})\end{array}$ & Mark $^{1}$ & Phase $^{2}$ \\
\hline $8 / 11 / 2002$ & $06 / 03$ & LSP & & & & & 106 & 2.0 & NO MARK & $\mathrm{U}$ \\
\hline $8 / 11 / 2002$ & $06 / 03$ & LSP & & & & & 137 & 4.6 & NO MARK & $\mathrm{U}$ \\
\hline $8 / 11 / 2002$ & $06 / 03$ & LSP & & & & & 149 & 5.1 & NO MARK & $\mathrm{U}$ \\
\hline $8 / 11 / 2002$ & $06 / 03$ & LSP & & & & & 134 & 4.0 & NO MARK & $\mathrm{U}$ \\
\hline $8 / 11 / 2002$ & $06 / 03$ & LSP & & & & & 102 & 2.1 & NO MARK & $\mathrm{U}$ \\
\hline $8 / 11 / 2002$ & $06 / 03$ & LSP & & & & & 139 & 4.4 & NO MARK & $\mathrm{U}$ \\
\hline $8 / 11 / 2002$ & $06 / 03$ & LSP & & & & & 95 & 1.5 & NO MARK & $\mathrm{U}$ \\
\hline $8 / 11 / 2002$ & $06 / 03$ & LSP & & & & & na & na & NO MARK & $\mathrm{U}$ \\
\hline $8 / 11 / 2002$ & $06 / 03$ & LSP & & & & & 126 & 3.1 & NO MARK & $\mathrm{U}$ \\
\hline $8 / 11 / 2002$ & $06 / 03$ & LSP & & & & & 147 & 5.1 & NO MARK & $\mathrm{U}$ \\
\hline $8 / 11 / 2002$ & $06 / 03$ & LSP & & & & & 137 & 4.3 & NO MARK & $\mathrm{U}$ \\
\hline $8 / 11 / 2002$ & $06 / 03$ & LSP & & & & & 134 & 4.1 & NO MARK & $\mathrm{U}$ \\
\hline $8 / 11 / 2002$ & $06 / 03$ & LSP & & & & & 142 & 4.7 & NO MARK & $\mathrm{U}$ \\
\hline $8 / 11 / 2002$ & $06 / 03$ & LSP & & & & & 125 & 2.8 & NO MARK & $\mathrm{U}$ \\
\hline $8 / 11 / 2002$ & $06 / 03$ & LSP & & & & & 95 & 1.6 & NO MARK & $\mathrm{U}$ \\
\hline $8 / 11 / 2002$ & $06 / 03$ & LSP & & & & & 132 & 3.7 & NO MARK & $\mathrm{U}$ \\
\hline $8 / 11 / 2002$ & $06 / 03$ & LSP & & & & & 105 & 2.0 & NO MARK & $\mathrm{U}$ \\
\hline $8 / 11 / 2002$ & $06 / 03$ & LSP & & & & & 104 & 1.9 & NO MARK & $\mathrm{U}$ \\
\hline $8 / 11 / 2002$ & $06 / 03$ & LSP & & & & & 111 & 2.3 & NO MARK & $\mathrm{U}$ \\
\hline $8 / 11 / 2002$ & $06 / 03$ & LSP & & & & & 103 & 1.9 & NO MARK & $\mathrm{U}$ \\
\hline $8 / 11 / 2002$ & $06 / 03$ & LSP & & & & & 104 & 1.8 & NO MARK & $\mathrm{U}$ \\
\hline $8 / 11 / 2002$ & $06 / 03$ & LSP & & & & & 119 & 2.6 & NO MARK & $\mathrm{U}$ \\
\hline $8 / 11 / 2002$ & $06 / 03$ & LSP & & & & & 132 & 3.9 & NO MARK & $\mathrm{U}$ \\
\hline $8 / 11 / 2002$ & $06 / 03$ & LSP & & & & & 101 & 1.7 & NO MARK & $\mathrm{U}$ \\
\hline $8 / 11 / 2002$ & $06 / 03$ & LSP & & & & & 97 & 1.3 & NO MARK & $\mathrm{U}$ \\
\hline $8 / 11 / 2002$ & $06 / 03$ & LSP & & & & & 115 & 2.4 & NO MARK & $\mathrm{U}$ \\
\hline $8 / 11 / 2002$ & $06 / 03$ & LSP & & & & & 118 & 2.4 & NO MARK & $\mathrm{U}$ \\
\hline $8 / 11 / 2002$ & $06 / 03$ & LSP & & & & & 100 & 1.5 & NO MARK & $\mathrm{U}$ \\
\hline $8 / 11 / 2002$ & $06 / 03$ & LSP & & & & & 102 & 1.6 & NO MARK & $\mathrm{U}$ \\
\hline $8 / 11 / 2002$ & $06 / 03$ & LSP & & & & & 102 & 1.6 & NO MARK & $\mathrm{U}$ \\
\hline $8 / 11 / 2002$ & $06 / 03$ & LSP & & & & & 112 & 2.2 & NO MARK & $\mathrm{U}$ \\
\hline $8 / 11 / 2002$ & $06 / 03$ & LSP & & & & & 103 & 2.0 & NO MARK & $\mathrm{U}$ \\
\hline $8 / 13 / 2002$ & $07 / 04$ & RIP & 165.4 & 193 & 9 & 8 & 132 & 3.8 & NO MARK & $\mathrm{U}$ \\
\hline $8 / 13 / 2002$ & 07/04 & RIP & & & & & 139 & 4.1 & NO MARK & $\mathrm{U}$ \\
\hline $8 / 13 / 2002$ & 07/04 & RIP & & & & & 155 & 5.7 & NO MARK & $\mathrm{U}$ \\
\hline $8 / 13 / 2002$ & 07/04 & RIP & & & & & 114 & 2.4 & NO MARK & $\mathrm{U}$ \\
\hline $8 / 13 / 2002$ & $07 / 04$ & RIP & & & & & 119 & 2.5 & NO MARK & $\mathrm{U}$ \\
\hline $8 / 13 / 2002$ & $07 / 04$ & RIP & & & & & 113 & 2.6 & NO MARK & $\mathrm{U}$ \\
\hline $8 / 13 / 2002$ & 07/04 & RIP & & & & & 128 & 3.4 & NO MARK & $\mathrm{U}$ \\
\hline $8 / 13 / 2002$ & $07 / 04$ & RIP & & & & & 128 & 3.5 & NO MARK & $\mathrm{U}$ \\
\hline $8 / 22 / 2002$ & $07 / 04$ & LSP & 106.9 & 156 & 5 & 5 & 130 & 3.5 & NO MARK & $\mathrm{U}$ \\
\hline $8 / 22 / 2002$ & 07/04 & LSP & & & & & 111 & 2.4 & NO MARK & $\mathrm{U}$ \\
\hline $8 / 22 / 2002$ & 07/04 & LSP & & & & & 153 & 5.8 & NO MARK & $\mathrm{U}$ \\
\hline $8 / 22 / 2002$ & $07 / 04$ & LSP & & & & & 142 & 4.8 & NO MARK & $\mathrm{U}$ \\
\hline $8 / 22 / 2002$ & $07 / 04$ & LSP & & & & & 129 & 3.4 & NO MARK & $\mathrm{U}$ \\
\hline
\end{tabular}

1 RCO-Right center orange

2 T-transformed; U-untransformed 
Table A1. (con't).

\begin{tabular}{|c|c|c|c|c|c|c|c|c|c|c|}
\hline $\begin{array}{c}\text { Date } \\
\text { Electrofished } \\
\end{array}$ & $\begin{array}{c}\mathrm{Km} / \\
\text { Reach }\end{array}$ & $\begin{array}{c}\text { Habitat } \\
\text { Type }\end{array}$ & $\begin{array}{c}\text { Area } \\
\text { Fished } \\
\left(\mathrm{m}^{2}\right)\end{array}$ & $\begin{array}{c}\text { Time } \\
\text { Fished } \\
\text { (Min.) }\end{array}$ & $\begin{array}{l}\text { Lamprey } \\
\text { Captured }\end{array}$ & $\begin{array}{c}\text { Lamprey } \\
\text { Measured }\end{array}$ & $\begin{array}{c}\text { Length } \\
(\mathrm{mm})\end{array}$ & $\begin{array}{c}\text { Weight } \\
(\mathrm{g})\end{array}$ & Mark $^{1}$ & Phase $^{2}$ \\
\hline $8 / 24 / 2002$ & $07 / 04$ & RIF & 104.0 & 204 & 2 & 0 & na & na & NO MARK & $\mathrm{U}$ \\
\hline $8 / 24 / 2002$ & $07 / 04$ & RIF & & & & & na & na & NO MARK & $\mathrm{U}$ \\
\hline $8 / 25 / 2002$ & $05 / 07$ & LSP & 196.5 & 356 & 36 & 34 & 123 & 2.8 & NO MARK & $\mathrm{U}$ \\
\hline $8 / 25 / 2002$ & 05/07 & LSP & & & & & 150 & 5.0 & NO MARK & $\mathrm{U}$ \\
\hline $8 / 25 / 2002$ & 05/07 & LSP & & & & & 139 & 3.8 & NO MARK & $\mathrm{U}$ \\
\hline $8 / 25 / 2002$ & 05/07 & LSP & & & & & 149 & 4.7 & NO MARK & $\mathrm{U}$ \\
\hline $8 / 25 / 2002$ & $05 / 07$ & LSP & & & & & 127 & 3.0 & NO MARK & $\mathrm{U}$ \\
\hline $8 / 25 / 2002$ & $05 / 07$ & LSP & & & & & 145 & 4.7 & NO MARK & $\mathrm{U}$ \\
\hline $8 / 25 / 2002$ & 05/07 & LSP & & & & & 129 & 3.5 & NO MARK & $\mathrm{U}$ \\
\hline $8 / 25 / 2002$ & 05/07 & LSP & & & & & 148 & 4.6 & NO MARK & $\mathrm{U}$ \\
\hline $8 / 25 / 2002$ & 05/07 & LSP & & & & & 135 & 4.0 & NO MARK & $\mathrm{U}$ \\
\hline $8 / 25 / 2002$ & $05 / 07$ & LSP & & & & & 116 & 2.5 & NO MARK & $\mathrm{U}$ \\
\hline $8 / 25 / 2002$ & $05 / 07$ & LSP & & & & & 149 & 5.0 & NO MARK & $\mathrm{U}$ \\
\hline $8 / 25 / 2002$ & $05 / 07$ & LSP & & & & & 134 & 3.9 & NO MARK & $\mathrm{U}$ \\
\hline $8 / 25 / 2002$ & $05 / 07$ & LSP & & & & & 143 & 4.7 & NO MARK & $\mathrm{U}$ \\
\hline $8 / 25 / 2002$ & $05 / 07$ & LSP & & & & & 123 & 2.7 & NO MARK & $\mathrm{U}$ \\
\hline $8 / 25 / 2002$ & $05 / 07$ & LSP & & & & & 127 & 3.3 & NO MARK & $\mathrm{U}$ \\
\hline $8 / 25 / 2002$ & $05 / 07$ & LSP & & & & & 110 & 2.3 & NO MARK & $\mathrm{U}$ \\
\hline $8 / 25 / 2002$ & $05 / 07$ & LSP & & & & & 140 & 4.3 & NO MARK & $\mathrm{U}$ \\
\hline $8 / 25 / 2002$ & $05 / 07$ & LSP & & & & & 123 & 3.0 & NO MARK & $\mathrm{U}$ \\
\hline $8 / 25 / 2002$ & $05 / 07$ & LSP & & & & & 148 & 5.5 & NO MARK & $\mathrm{U}$ \\
\hline $8 / 25 / 2002$ & $05 / 07$ & LSP & & & & & 124 & 3.1 & NO MARK & $\mathrm{U}$ \\
\hline $8 / 25 / 2002$ & 05/07 & LSP & & & & & 127 & 3.3 & NO MARK & $\mathrm{U}$ \\
\hline $8 / 25 / 2002$ & 05/07 & LSP & & & & & 148 & 4.6 & NO MARK & $\mathrm{U}$ \\
\hline $8 / 25 / 2002$ & 05/07 & LSP & & & & & 119 & 2.8 & NO MARK & $\mathrm{U}$ \\
\hline $8 / 25 / 2002$ & 05/07 & LSP & & & & & 126 & 3.2 & NO MARK & $\mathrm{U}$ \\
\hline $8 / 25 / 2002$ & $05 / 07$ & LSP & & & & & 125 & 3.1 & NO MARK & $\mathrm{U}$ \\
\hline $8 / 25 / 2002$ & $05 / 07$ & LSP & & & & & 125 & 3.2 & NO MARK & $\mathrm{U}$ \\
\hline $8 / 25 / 2002$ & $05 / 07$ & LSP & & & & & 115 & 2.9 & NO MARK & $\mathrm{U}$ \\
\hline $8 / 25 / 2002$ & $05 / 07$ & LSP & & & & & 105 & 2.0 & NO MARK & $\mathrm{U}$ \\
\hline $8 / 25 / 2002$ & 05/07 & LSP & & & & & 119 & 2.8 & NO MARK & $\mathrm{U}$ \\
\hline $8 / 25 / 2002$ & 05/07 & LSP & & & & & 131 & 3.7 & NO MARK & $\mathrm{U}$ \\
\hline $8 / 25 / 2002$ & 05/07 & LSP & & & & & 117 & 2.6 & NO MARK & $\mathrm{U}$ \\
\hline $8 / 25 / 2002$ & $05 / 07$ & LSP & & & & & 122 & 2.9 & NO MARK & $\mathrm{U}$ \\
\hline $8 / 25 / 2002$ & $05 / 07$ & LSP & & & & & 124 & 3.1 & NO MARK & $\mathrm{U}$ \\
\hline $8 / 25 / 2002$ & $05 / 07$ & LSP & & & & & 114 & 2.1 & NO MARK & $\mathrm{U}$ \\
\hline $8 / 27 / 2002$ & $05 / 07$ & RIF & 78.0 & 72 & 10 & 10 & 125 & 3.1 & NO MARK & $\mathrm{U}$ \\
\hline $8 / 27 / 2002$ & $05 / 07$ & RIF & & & & & 140 & 4.4 & NO MARK & $\mathrm{U}$ \\
\hline $8 / 27 / 2002$ & $05 / 07$ & RIF & & & & & 147 & 4.7 & NO MARK & $\mathrm{U}$ \\
\hline $8 / 27 / 2002$ & $05 / 07$ & RIF & & & & & 124 & 2.9 & NO MARK & $\mathrm{U}$ \\
\hline $8 / 27 / 2002$ & 05/07 & RIF & & & & & 118 & 2.9 & NO MARK & $\mathrm{U}$ \\
\hline $8 / 27 / 2002$ & 05/07 & RIF & & & & & 117 & 2.4 & NO MARK & $\mathrm{U}$ \\
\hline $8 / 27 / 2002$ & 05/07 & RIF & & & & & 144 & 4.7 & NO MARK & $\mathrm{U}$ \\
\hline $8 / 27 / 2002$ & 05/07 & RIF & & & & & 121 & 2.7 & NO MARK & $\mathrm{U}$ \\
\hline $8 / 27 / 2002$ & 05/07 & RIF & & & & & 126 & 2.9 & NO MARK & $\mathrm{U}$ \\
\hline $8 / 27 / 2002$ & $05 / 07$ & RIF & & & & & 153 & 5.6 & NO MARK & $\mathrm{U}$ \\
\hline
\end{tabular}

1. $\mathrm{RCO}$ - right center orange

2. T- transformed; U- untransformed 
Table A2. Pacific lamprey length, weight, and mark information for S.F.Clearwater River, ID, 2002.

\begin{tabular}{|c|c|c|c|c|c|c|c|c|c|c|}
\hline $\begin{array}{c}\text { Date } \\
\text { Electrofished } \\
\end{array}$ & $\begin{array}{c}\mathrm{Km} / \\
\text { Reach }\end{array}$ & $\begin{array}{c}\text { Habitat } \\
\text { Type }\end{array}$ & $\begin{array}{c}\text { Area } \\
\text { Fished } \\
\left(\mathrm{m}^{2}\right) \\
\end{array}$ & $\begin{array}{c}\text { Time } \\
\text { Fished } \\
(\text { Min })\end{array}$ & $\begin{array}{l}\text { Lamprey } \\
\text { Captured }\end{array}$ & $\begin{array}{l}\text { Lamprey } \\
\text { Measured }\end{array}$ & $\begin{array}{c}\text { Length } \\
(\mathrm{mm})\end{array}$ & $\begin{array}{c}\text { Weight } \\
(\mathrm{g})\end{array}$ & Mark $^{1}$ & Phase $^{2}$ \\
\hline $9 / 5 / 2002$ & 49/na & LSP & $\sim 10.0$ & 12 & 44 & 44 & 145 & 4.8 & NO MARK & $\mathrm{U}$ \\
\hline $9 / 5 / 2002$ & 49/na & LSP & & & & & 142 & 4.5 & NO MARK & $\mathrm{U}$ \\
\hline $9 / 5 / 2002$ & 49/na & LSP & & & & & 134 & 4.3 & NO MARK & $\mathrm{U}$ \\
\hline $9 / 5 / 2002$ & 49/na & LSP & & & & & 104 & 1.5 & NO MARK & $\mathrm{U}$ \\
\hline $9 / 5 / 2002$ & 49/na & LSP & & & & & 105 & 1.8 & NO MARK & $\mathrm{U}$ \\
\hline $9 / 5 / 2002$ & 49/na & LSP & & & & & 135 & 2.5 & NO MARK & $\mathrm{U}$ \\
\hline $9 / 5 / 2002$ & 49/na & LSP & & & & & 143 & 4.4 & NO MARK & $\mathrm{U}$ \\
\hline $9 / 5 / 2002$ & 49/na & LSP & & & & & 95 & -- & NO MARK & $\mathrm{U}$ \\
\hline $9 / 5 / 2002$ & 49/na & LSP & & & & & 139 & 4.1 & NO MARK & $\mathrm{U}$ \\
\hline $9 / 5 / 2002$ & 49/na & LSP & & & & & 128 & 4.0 & NO MARK & $\mathrm{U}$ \\
\hline $9 / 5 / 2002$ & 49/na & LSP & & & & & 120 & -- & NO MARK & $\mathrm{U}$ \\
\hline $9 / 5 / 2002$ & 49/na & LSP & & & & & 121 & 3.2 & NO MARK & $\mathrm{U}$ \\
\hline $9 / 5 / 2002$ & 49/na & LSP & & & & & 142 & -- & NO MARK & $\mathrm{U}$ \\
\hline $9 / 5 / 2002$ & 49/na & LSP & & & & & 109 & 1.9 & NO MARK & $\mathrm{U}$ \\
\hline $9 / 5 / 2002$ & 49/na & LSP & & & & & 112 & 2.0 & NO MARK & $\mathrm{U}$ \\
\hline $9 / 5 / 2002$ & 49/na & LSP & & & & & 134 & 3.8 & NO MARK & $\mathrm{U}$ \\
\hline $9 / 5 / 2002$ & 49/na & LSP & & & & & 120 & 2.5 & NO MARK & $\mathrm{U}$ \\
\hline $9 / 5 / 2002$ & 49/na & LSP & & & & & 122 & 2.6 & NO MARK & $\mathrm{U}$ \\
\hline $9 / 5 / 2002$ & 49/na & LSP & & & & & 97 & 1.3 & NO MARK & $\mathrm{U}$ \\
\hline $9 / 5 / 2002$ & 49/na & LSP & & & & & 119 & 2.1 & NO MARK & $\mathrm{U}$ \\
\hline $9 / 5 / 2002$ & 49/na & LSP & & & & & 103 & 1.1 & NO MARK & $\mathrm{U}$ \\
\hline $9 / 5 / 2002$ & 49/na & LSP & & & & & 92 & 0.9 & NO MARK & $\mathrm{U}$ \\
\hline $9 / 5 / 2002$ & 49/na & LSP & & & & & 100 & 1.2 & NO MARK & $\mathrm{U}$ \\
\hline $9 / 5 / 2002$ & 49/na & LSP & & & & & 129 & 3.0 & NO MARK & $\mathrm{U}$ \\
\hline $9 / 5 / 2002$ & 49/na & LSP & & 8 & & & 130 & 2.6 & NO MARK & $\mathrm{U}$ \\
\hline $9 / 5 / 2002$ & 49/na & LSP & & & & & 137 & 4.0 & NO MARK & $\mathrm{U}$ \\
\hline $9 / 5 / 2002$ & 49/na & LSP & & & & & 116 & 2.7 & NO MARK & $\mathrm{U}$ \\
\hline $9 / 5 / 2002$ & 49/na & LSP & & & & & 125 & 3.5 & NO MARK & U \\
\hline $9 / 5 / 2002$ & 49/na & LSP & & & & & 115 & 2.2 & NO MARK & $\mathrm{U}$ \\
\hline $9 / 5 / 2002$ & 49/na & LSP & & & & & 141 & 4.7 & NO MARK & $\mathrm{U}$ \\
\hline $9 / 5 / 2002$ & 49/na & LSP & & & & & 100 & 1.6 & NO MARK & $\mathrm{U}$ \\
\hline $9 / 5 / 2002$ & 49/na & LSP & & & & & 132 & 3.8 & NO MARK & $\mathrm{U}$ \\
\hline $9 / 5 / 2002$ & 49/na & LSP & & & & & 142 & 4.5 & NO MARK & $\mathrm{U}$ \\
\hline $9 / 5 / 2002$ & 49/na & LSP & & & & & 107 & 1.6 & NO MARK & $\mathrm{U}$ \\
\hline $9 / 5 / 2002$ & 49/na & LSP & & & & & 116 & 2.6 & NO MARK & $\mathrm{U}$ \\
\hline $9 / 5 / 2002$ & 49/na & LSP & & & & & 139 & 3.2 & NO MARK & $\mathrm{U}$ \\
\hline $9 / 5 / 2002$ & 49/na & LSP & & & & & 123 & 2.8 & NO MARK & $\mathrm{U}$ \\
\hline $9 / 5 / 2002$ & 49/na & LSP & & & & & 118 & 2.8 & NO MARK & $\mathrm{U}$ \\
\hline $9 / 5 / 2002$ & 49/na & LSP & & & & & 123 & 2.9 & NO MARK & $\mathrm{U}$ \\
\hline $9 / 5 / 2002$ & 49/na & LSP & & & & & 117 & 2.4 & NO MARK & $\mathrm{U}$ \\
\hline $9 / 5 / 2002$ & 49/na & LSP & & & & & 116 & 2.8 & NO MARK & $\mathrm{U}$ \\
\hline $9 / 5 / 2002$ & 49/na & LSP & & & & & 87 & 0.9 & NO MARK & U \\
\hline $9 / 5 / 2002$ & 49/na & LSP & & & & & 91 & 1.0 & NO MARK & $\mathrm{U}$ \\
\hline $9 / 5 / 2002$ & 49/na & LSP & & & & & 89 & 1.0 & NO MARK & $\mathrm{U}$ \\
\hline
\end{tabular}

1 RCO-Right center orange

2 T-transformed; U-untransformed 
Table A2. (con't).

Area Time

Date $\mathrm{Km} /$ Habitat Fished Fished Lamprey Lamprey Length Weight

\begin{tabular}{cccccccccccc} 
Electrofished & Reach & Type & $\left(\mathrm{m}^{2}\right)$ & $($ Min $)$ & Captured Measured & $(\mathrm{mm})$ & $(\mathrm{g})$ & Mark $^{1}$ & Phase $^{2}$ \\
\hline 9/6/2002 & $40 / \mathrm{na}$ & LSP & 10.0 & 13 & 63 & 63 & 92 & 1.2 & NO MARK & U
\end{tabular}

$\begin{array}{lllllllllll}9 / 6 / 2002 & 40 / n a & \text { LSP } & 10.0 & 13 & 63 & 63 & 92 & 1.2 & \text { NO MARK } & \text { U } \\ 9 / 6 / 2002 & 40 / n a & \text { LSP } & & & & & 95 & 1.6 & \text { NO MARK } & \text { U }\end{array}$

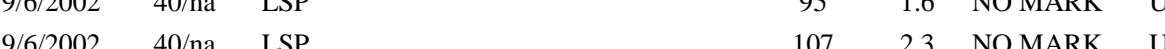

$\begin{array}{lllllll}9 / 6 / 2002 & 40 / n a & \text { LSP } & 117 & 2.8 & \text { NO MARK } & \text { U }\end{array}$

9/6/2002 40/na LSP $\quad 88 \quad 1.0 \quad$ NO MARK U

9/6/2002 40/na LSP $\quad 110 \quad 2.5$ NO MARK U

9/6/2002 40/na LSP $\quad 95 \quad 1.4$ NO MARK U

9/6/2002 40/na LSP $\quad 101 \quad 2.0 \quad$ NO MARK U

9/6/2002 40/na LSP $\quad 94 \quad--\quad$ NO MARK U

$\begin{array}{llllllll}9 / 6 / 2002 & 40 / \text { na } & \text { LSP } & 100 & 2.1 & \text { NO MARK } & \text { U }\end{array}$

9/6/2002 40/na LSP $\quad 120 \quad 2.6 \quad$ NO MARK U

9/6/2002 40/na LSP $\quad 103 \quad 1.9$ NO MARK U

9/6/2002 40/na LSP $\quad 92 \quad 1.3$ NO MARK U

$\begin{array}{lllllll}9 / 6 / 2002 & 40 / \text { na } & \text { LSP } & 122 & 2.8 & \text { NO MARK } & \text { U }\end{array}$

9/6/2002 40/na LSP $\quad 111 \quad 2.0 \quad$ NO MARK U

$\begin{array}{lllllll}9 / 6 / 2002 & 40 / \text { na } & \text { LSP } & 93 & 1.4 & \text { NO MARK } & \text { U }\end{array}$

9/6/2002 40/na LSP $\quad 104 \quad 1.8$ NO MARK U

9/6/2002 40/na LSP $\quad 105 \quad 2.0 \quad$ NO MARK U

9/6/2002 40/na LSP $\quad 112 \quad 2.4$ NO MARK $\quad$ U

9/6/2002 40/na LSP $\quad 125 \quad 3.1$ NO MARK $\quad$ U

9/6/2002 40/na LSP $\quad 77 \quad 0.8$ NO MARK U

9/6/2002 40/na LSP $124 \quad 2.5$ NO MARK U

9/6/2002 40/na LSP $\quad 128 \quad--$ NO MARK U

9/6/2002 40/na LSP $\quad 97 \quad--\quad$ NO MARK U

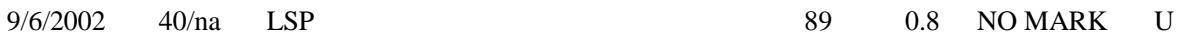

9/6/2002 40/na LSP $\quad 95 \quad--\quad$ NO MARK U

$\begin{array}{lllllll}9 / 6 / 2002 & 40 / \text { na } & \text { LSP } & 104 & 1.6 & \text { NO MARK } & \text { U }\end{array}$

9/6/2002 40/na LSP $\quad 89 \quad 1.4$ NO MARK U

9/6/2002 40/na LSP $\quad 111 \quad 2.3$ NO MARK U

9/6/2002 40/na LSP $\quad 92 \quad--\quad$ NO MARK U

9/6/2002 40/na LSP $\quad 95 \quad 1.7$ NO MARK U

$\begin{array}{llllllll}9 / 6 / 2002 & 40 / \mathrm{na} & \text { LSP } & 92 & 1.4 & \text { NO MARK } & \text { U }\end{array}$

$\begin{array}{lllllll}9 / 6 / 2002 & 40 / \text { na } & \text { LSP } & 107 & 2.0 & \text { NO MARK } & \text { U }\end{array}$

9/6/2002 40/na LSP $\quad 85 \quad 1.2$ NO MARK U

$\begin{array}{lllllll}9 / 6 / 2002 & 40 / \text { na } & \text { LSP } & 112 & 2.5 & \text { NO MARK } & \text { U }\end{array}$

9/6/2002 40/na LSP $114 \quad 2.8$ NO MARK U

$\begin{array}{lllllll}9 / 6 / 2002 & 40 / \text { na } & \text { LSP } & 98 & 1.7 & \text { NO MARK } & \text { U }\end{array}$

$\begin{array}{lllllll}9 / 6 / 2002 & 40 / \text { na } & \text { LSP } & 108 & 1.8 & \text { NO MARK } & \text { U }\end{array}$

9/6/2002 40/na LSP $104 \quad--$ NO MARK U

$\begin{array}{llllllll}9 / 6 / 2002 & 40 / \text { na } & \text { LSP } & 91 & 1.0 & \text { NO MARK } & \text { U }\end{array}$

9/6/2002 40/na LSP $4117 \quad--\quad$ NO MARK U

9/6/2002 40/na LSP $\quad 98 \quad 1.6 \quad$ NO MARK U

9/6/2002 40/na LSP $109 \quad 2.2$ NO MARK U

9/6/2002 40/na LSP $\quad 89 \quad--\quad$ NO MARK U

9/6/2002 40/na LSP $111 \quad--$ NO MARK U

1 RCO-Right center orange

2 T-transformed; U-untransformed 
Table A2. (con't).

$$
\text { Area Time }
$$

Date Km/ Habitat Fished Fished Lamprey Lamprey Length Weight

\begin{tabular}{|c|c|c|c|c|c|c|c|c|c|}
\hline Electrofished & Reach & Type & $\left(\mathrm{m}^{2}\right)$ & $(\operatorname{Min})$ & Captured Measured & $(\mathrm{mm})$ & $(\mathrm{g})$ & $\operatorname{Mark}^{1}$ & Phase $^{2}$ \\
\hline $9 / 6 / 2002$ & 40/na & LSP & & & & 100 & 2.0 & NO MARK & $\mathrm{U}$ \\
\hline $9 / 6 / 2002$ & 40/na & LSP & & & & 102 & -- & NO MARK & $\mathrm{U}$ \\
\hline $9 / 6 / 2002$ & 40/na & LSP & & & & 105 & -- & NO MARK & $\mathrm{U}$ \\
\hline $9 / 6 / 2002$ & 40/na & LSP & & & & 91 & -- & NO MARK & $\mathrm{U}$ \\
\hline $9 / 6 / 2002$ & 40/na & LSP & & & & 114 & -- & NO MARK & $\mathrm{U}$ \\
\hline $9 / 6 / 2002$ & 40/na & LSP & & & & 119 & -- & NO MARK & $\mathrm{U}$ \\
\hline $9 / 6 / 2002$ & 40/na & LSP & & & & 105 & -- & NO MARK & $\mathrm{U}$ \\
\hline $9 / 6 / 2002$ & 40/na & LSP & & & & 101 & -- & NO MARK & $\mathrm{U}$ \\
\hline $9 / 6 / 2002$ & 40/na & LSP & & & & 113 & -- & NO MARK & $\mathrm{U}$ \\
\hline $9 / 6 / 2002$ & 40/na & LSP & & & & 101 & -- & NO MARK & $\mathrm{U}$ \\
\hline $9 / 6 / 2002$ & 40/na & LSP & & & & 118 & -- & NO MARK & $\mathrm{U}$ \\
\hline $9 / 6 / 2002$ & 40/na & LSP & & & & 92 & -- & NO MARK & $\mathrm{U}$ \\
\hline $9 / 6 / 2002$ & 40/na & LSP & & & & 91 & -- & NO MARK & $\mathrm{U}$ \\
\hline $9 / 6 / 2002$ & 40/na & LSP & & & & 92 & -- & NO MARK & $\mathrm{U}$ \\
\hline $9 / 6 / 2002$ & 40/na & LSP & & & & 110 & -- & NO MARK & $\mathrm{U}$ \\
\hline $9 / 6 / 2002$ & 40/na & LSP & & & & 110 & -- & NO MARK & $\mathrm{U}$ \\
\hline $9 / 6 / 2002$ & 40/na & LSP & & & & 96 & -- & NO MARK & $\mathrm{U}$ \\
\hline $9 / 6 / 2002$ & 40/na & LSP & & & & 123 & -- & NO MARK & $\mathrm{U}$ \\
\hline
\end{tabular}

1 RCO-Right center orange

2 T-transformed; U-untransformed 
Table A3. Pacific lamprey length, weight, and mark information for Selway

River, ID, 2002.

\begin{tabular}{|c|c|c|c|c|c|c|c|c|c|c|}
\hline $\begin{array}{c}\text { Date } \\
\text { Electrofished }\end{array}$ & $\begin{array}{c}\mathrm{Km} / \\
\text { Reach }\end{array}$ & $\begin{array}{c}\text { Habitat } \\
\text { Type }\end{array}$ & $\begin{array}{c}\text { Area } \\
\text { Fished } \\
\left(\mathrm{m}^{2}\right)\end{array}$ & $\begin{array}{c}\text { Time } \\
\text { Fished } \\
\text { (Min.) }\end{array}$ & $\begin{array}{l}\text { Lamprey } \\
\text { Captured }\end{array}$ & $\begin{array}{l}\text { Lamprey } \\
\text { Measured }\end{array}$ & $\begin{array}{c}\text { Length } \\
(\mathrm{mm})\end{array}$ & $\begin{array}{c}\text { Weight } \\
(\mathrm{g})\end{array}$ & Mark $^{1}$ & Phase $^{2}$ \\
\hline $9 / 7 / 2002$ & 150m up frm. Gedney Crk. & LSP & 5.0 & 16 & 16 & 16 & 50 & 0.3 & NO MARK & $\mathrm{U}$ \\
\hline $9 / 7 / 2002$ & 150m up frm. Gedney Crk. & LSP & & & & & 43 & 0.3 & NO MARK & $\mathrm{U}$ \\
\hline $9 / 7 / 2002$ & 150m up frm. Gedney Crk. & LSP & & & & & 59 & 0.4 & NO MARK & $\mathrm{U}$ \\
\hline $9 / 7 / 2002$ & 150m up frm. Gedney Crk. & LSP & & & & & 49 & 0.3 & NO MARK & $\mathrm{U}$ \\
\hline $9 / 7 / 2002$ & 150m up frm. Gedney Crk. & LSP & & & & & 52 & 0.3 & NO MARK & $\mathrm{U}$ \\
\hline $9 / 7 / 2002$ & 150m up frm. Gedney Crk. & LSP & & & & & 82 & 0.8 & NO MARK & $\mathrm{U}$ \\
\hline $9 / 7 / 2002$ & 150m up frm. Gedney Crk. & LSP & & & & & 112 & 2.0 & NO MARK & $\mathrm{U}$ \\
\hline $9 / 7 / 2002$ & 150m up frm. Gedney Crk. & LSP & & & & & 77 & 0.8 & NO MARK & $\mathrm{U}$ \\
\hline $9 / 7 / 2002$ & 150m up frm. Gedney Crk. & LSP & & & & & 82 & 0.9 & NO MARK & $\mathrm{U}$ \\
\hline $9 / 7 / 2002$ & 150m up frm. Gedney Crk. & LSP & & & & & 75 & 0.8 & NO MARK & $\mathrm{U}$ \\
\hline $9 / 7 / 2002$ & 150m up frm. Gedney Crk. & LSP & & & & & 75 & 0.7 & NO MARK & $\mathrm{U}$ \\
\hline $9 / 7 / 2002$ & 150m up frm. Gedney Crk. & LSP & & & & & 84 & 0.9 & NO MARK & $\mathrm{U}$ \\
\hline $9 / 7 / 2002$ & 150m up frm. Gedney Crk. & LSP & & & & & 78 & 0.8 & NO MARK & $\mathrm{U}$ \\
\hline $9 / 7 / 2002$ & 150m up frm. Gedney Crk. & LSP & & & & & 90 & 1.3 & NO MARK & $\mathrm{U}$ \\
\hline $9 / 7 / 2002$ & 150m up frm. Gedney Crk. & LSP & & & & & 86 & 1.0 & NO MARK & $\mathrm{U}$ \\
\hline $9 / 7 / 2002$ & 150m up frm. Gedney Crk. & LSP & & & & & 69 & 0.7 & NO MARK & $\mathrm{U}$ \\
\hline $9 / 8 / 2002$ & 100m up frm. Jnsn. Bar & LSP & 5.0 & 13 & 45 & 45 & 52 & 0.3 & NO MARK & $\mathrm{U}$ \\
\hline $9 / 8 / 2002$ & 100m up frm. Jnsn. Bar & LSP & & & & & 54 & -- & NO MARK & $\mathrm{U}$ \\
\hline $9 / 8 / 2002$ & 100m up frm. Jnsn. Bar & LSP & & & & & 58 & 0.4 & NO MARK & $\mathrm{U}$ \\
\hline $9 / 8 / 2002$ & 100m up frm. Jnsn. Bar & LSP & & & & & 61 & 0.5 & NO MARK & U \\
\hline $9 / 8 / 2002$ & 100m up frm. Jnsn. Bar & LSP & & & & & 49 & 0.2 & NO MARK & U \\
\hline $9 / 8 / 2002$ & 100m up frm. Jnsn. Bar & LSP & & & & & 58 & 0.4 & NO MARK & $\mathrm{U}$ \\
\hline $9 / 8 / 2002$ & 100m up frm. Jnsn. Bar & LSP & & & & & 94 & 1.2 & NO MARK & U \\
\hline $9 / 8 / 2002$ & 100m up frm. Jnsn. Bar & LSP & & & & & 51 & 0.4 & NO MARK & $\mathrm{U}$ \\
\hline $9 / 8 / 2002$ & 100m up frm. Jnsn. Bar & LSP & & & & & 61 & 0.6 & NO MARK & $\mathrm{U}$ \\
\hline $9 / 8 / 2002$ & 100m up frm. Jnsn. Bar & LSP & & & & & 56 & -- & NO MARK & $\mathrm{U}$ \\
\hline $9 / 8 / 2002$ & 100m up frm. Jnsn. Bar & LSP & & & & & 56 & -- & NO MARK & $\mathrm{U}$ \\
\hline $9 / 8 / 2002$ & 100m up frm. Jnsn. Bar & LSP & & & & & 59 & -- & NO MARK & $\mathrm{U}$ \\
\hline $9 / 8 / 2002$ & 100m up frm. Jnsn. Bar & LSP & & & & & 55 & 0.4 & NO MARK & $\mathrm{U}$ \\
\hline $9 / 8 / 2002$ & 100m up frm. Jnsn. Bar & LSP & & & & & 57 & 0.4 & NO MARK & $\mathrm{U}$ \\
\hline $9 / 8 / 2002$ & 100m up frm. Jnsn. Bar & LSP & & & & & 47 & 0.2 & NO MARK & $\mathrm{U}$ \\
\hline $9 / 8 / 2002$ & 100m up frm. Jnsn. Bar & LSP & & & & & 97 & 1.5 & NO MARK & $\mathrm{U}$ \\
\hline $9 / 8 / 2002$ & 100m up frm. Jnsn. Bar & LSP & & & & & 125 & 2.7 & NO MARK & $\mathrm{U}$ \\
\hline $9 / 8 / 2002$ & 100m up frm. Jnsn. Bar & LSP & & & & & 111 & 1.7 & NO MARK & $\mathrm{U}$ \\
\hline $9 / 8 / 2002$ & 100m up frm. Jnsn. Bar & LSP & & & & & 116 & -- & NO MARK & $\mathrm{U}$ \\
\hline $9 / 8 / 2002$ & 100m up frm. Jnsn. Bar & LSP & & & & & 105 & -- & NO MARK & $\mathrm{U}$ \\
\hline $9 / 8 / 2002$ & 100m up frm. Jnsn. Bar & LSP & & & & & 105 & 1.9 & NO MARK & U \\
\hline $9 / 8 / 2002$ & 100m up frm. Jnsn. Bar & LSP & & & & & 99 & -- & NO MARK & $\mathrm{U}$ \\
\hline $9 / 8 / 2002$ & 100m up frm. Jnsn. Bar & LSP & & & & & 100 & 1.7 & NO MARK & U \\
\hline $9 / 8 / 2002$ & 100m up frm. Jnsn. Bar & LSP & & & & & 122 & 2.7 & NO MARK & $\mathrm{U}$ \\
\hline $9 / 8 / 2002$ & 100m up frm. Jnsn. Bar & LSP & & & & & 107 & 1.7 & NO MARK & $\mathrm{U}$ \\
\hline
\end{tabular}

1 RCO-Right center orange

2 T-transformed; U-untransformed 
Table A3. (con't).

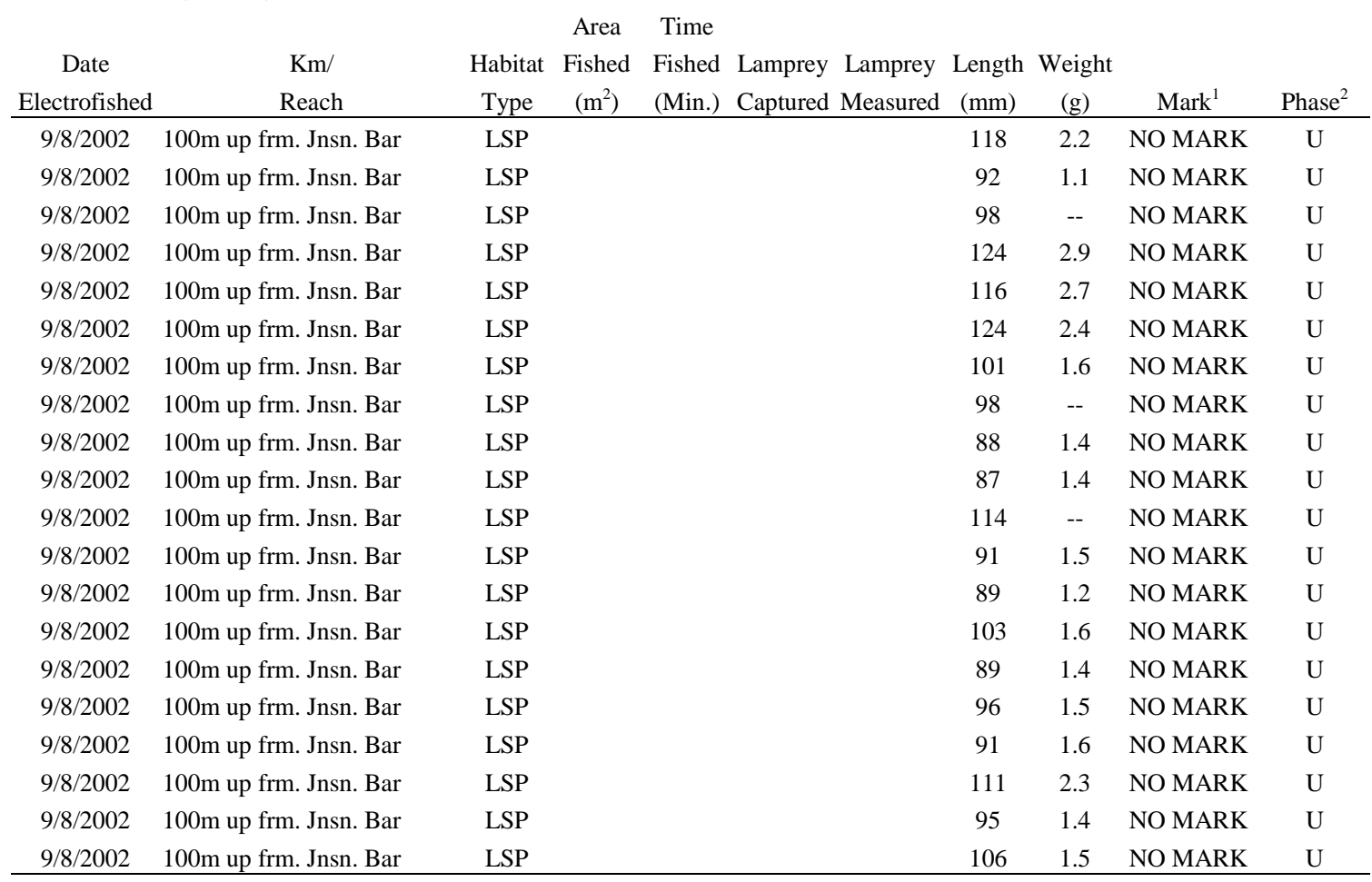

1. RCO-Right center orange

2. T-transformed; U-untransformed 
Table A4. Pacific lamprey length, weight, and mark information for Lochsa

River, ID, 2002.

\begin{tabular}{|c|c|c|c|c|c|c|c|c|c|c|}
\hline $\begin{array}{c}\text { Date } \\
\text { Electrofished }\end{array}$ & $\begin{array}{c}\mathrm{Km} / \\
\text { Reach }\end{array}$ & $\begin{array}{r}\text { Habitat } \\
\text { Type } \\
\end{array}$ & $\begin{array}{r}\text { Area } \\
\text { Fished } \\
\left(\mathrm{m}^{2}\right)\end{array}$ & $\begin{array}{c}\text { Time } \\
\text { Fished } \\
\text { (Min.) }\end{array}$ & $\begin{array}{l}\text { Lamprey } \\
\text { Captured }\end{array}$ & $\begin{array}{l}\text { Lamprey } \\
\text { Measured }\end{array}$ & $\begin{array}{c}\text { Length } \\
(\mathrm{mm})\end{array}$ & $\begin{array}{c}\text { Weight } \\
(\mathrm{g})\end{array}$ & Mark $^{1}$ & Phase $^{2}$ \\
\hline $7 / 26 / 2002$ & $.2 \mathrm{~km}$ dwn. Gngr. Crk. & LSP & 5.0 & 9 & 4 & 4 & $\sim>100,<180$ & -- & NO MARK & $\mathrm{U}$ \\
\hline $7 / 26 / 2002$ & $.2 \mathrm{~km}$ dwn. Gngr. Crk. & LSP & & & & & $\sim>100,<180$ & -- & NO MARK & $\mathrm{U}$ \\
\hline $7 / 26 / 2002$ & $.2 \mathrm{~km}$ dwn. Gngr. Crk. & LSP & & & & & $\sim>100,<180$ & -- & NO MARK & $\mathrm{U}$ \\
\hline $7 / 26 / 2002$ & $.2 \mathrm{~km}$ dwn. Gngr. Crk. & LSP & & & & & $\sim>100,<180$ & -- & NO MARK & $\mathrm{U}$ \\
\hline $7 / 27 / 2002$ & $.1 \mathrm{~km}$ up Castle Crk. & LSP & 5.0 & 12.5 & 12 & 12 & 118 & 2.9 & NO MARK & $\mathrm{U}$ \\
\hline $7 / 27 / 2002$ & $.1 \mathrm{~km}$ up Castle Crk. & LSP & & & & & 96 & 1.7 & NO MARK & $\mathrm{U}$ \\
\hline $7 / 27 / 2002$ & $.1 \mathrm{~km}$ up Castle Crk. & LSP & & & & & 107 & 2.7 & NO MARK & $\mathrm{U}$ \\
\hline $7 / 27 / 2002$ & $.1 \mathrm{~km}$ up Castle Crk. & LSP & & & & & 75 & 1.4 & NO MARK & $\mathrm{U}$ \\
\hline $7 / 27 / 2002$ & $.1 \mathrm{~km}$ up Castle Crk. & LSP & & & & & 109 & 2.3 & NO MARK & $\mathrm{U}$ \\
\hline $7 / 27 / 2002$ & $.1 \mathrm{~km}$ up Castle Crk. & LSP & & & & & 102 & 2.4 & NO MARK & $\mathrm{U}$ \\
\hline $7 / 27 / 2002$ & $.1 \mathrm{~km}$ up Castle Crk. & LSP & & & & & 53 & -- & NO MARK & $\mathrm{U}$ \\
\hline $7 / 27 / 2002$ & $.1 \mathrm{~km}$ up Castle Crk. & LSP & & & & & 116 & 2.2 & NO MARK & $\mathrm{U}$ \\
\hline $7 / 27 / 2002$ & $.1 \mathrm{~km}$ up Castle Crk. & LSP & & & & & 117 & -- & NO MARK & $\mathrm{U}$ \\
\hline $7 / 27 / 2002$ & $.1 \mathrm{~km}$ up Castle Crk. & LSP & & & & & 88 & -- & NO MARK & U \\
\hline $7 / 27 / 2002$ & $.1 \mathrm{~km}$ up Castle Crk. & LSP & & & & & 109 & 2.4 & NO MARK & $\mathrm{U}$ \\
\hline $7 / 27 / 2002$ & $.1 \mathrm{~km}$ up Castle Crk. & LSP & & & & & 90 & 2.0 & NO MARK & $\mathrm{U}$ \\
\hline $7 / 28 / 2002$ & $.1 \mathrm{~km}$ dwn. Fire Crk. & LSP & 2.0 & 11 & 38 & 38 & 105 & 1.9 & NO MARK & $\mathrm{U}$ \\
\hline $7 / 28 / 2002$ & $.1 \mathrm{~km}$ dwn. Fire Crk. & LSP & & & & & 120 & 3.0 & NO MARK & $\mathrm{U}$ \\
\hline $7 / 28 / 2002$ & $.1 \mathrm{~km}$ dwn. Fire Crk. & LSP & & & & & 79 & 0.9 & NO MARK & $\mathrm{U}$ \\
\hline $7 / 28 / 2002$ & $.1 \mathrm{~km}$ dwn. Fire Crk. & LSP & & & & & 63 & 0.6 & NO MARK & $\mathrm{U}$ \\
\hline $7 / 28 / 2002$ & $.1 \mathrm{~km}$ dwn. Fire Crk. & LSP & & & & & 71 & 0.7 & NO MARK & $\mathrm{U}$ \\
\hline $7 / 28 / 2002$ & $.1 \mathrm{~km}$ dwn. Fire Crk. & LSP & & & & & 63 & 0.7 & NO MARK & $\mathrm{U}$ \\
\hline $7 / 28 / 2002$ & $.1 \mathrm{~km}$ dwn. Fire Crk. & LSP & & & & & 105 & 2.1 & NO MARK & U \\
\hline $7 / 28 / 2002$ & $.1 \mathrm{~km}$ dwn. Fire Crk. & LSP & & & & & 71 & 0.9 & NO MARK & $\mathrm{U}$ \\
\hline $7 / 28 / 2002$ & $.1 \mathrm{~km}$ dwn. Fire Crk. & LSP & & & & & 99 & 1.7 & NO MARK & $\mathrm{U}$ \\
\hline $7 / 28 / 2002$ & $.1 \mathrm{~km}$ dwn. Fire Crk. & LSP & & & & & 75 & 0.9 & NO MARK & $\mathrm{U}$ \\
\hline $7 / 28 / 2002$ & $.1 \mathrm{~km}$ dwn. Fire Crk. & LSP & & & & & 104 & 1.9 & NO MARK & $\mathrm{U}$ \\
\hline $7 / 28 / 2002$ & $.1 \mathrm{~km}$ dwn. Fire Crk. & LSP & & & & & 46 & 0.2 & NO MARK & $\mathrm{U}$ \\
\hline $7 / 28 / 2002$ & $.1 \mathrm{~km}$ dwn. Fire Crk. & LSP & & & & & 65 & 0.7 & NO MARK & $\mathrm{U}$ \\
\hline $7 / 28 / 2002$ & $.1 \mathrm{~km}$ dwn. Fire Crk. & LSP & & & & & 68 & 0.8 & NO MARK & $\mathrm{U}$ \\
\hline $7 / 28 / 2002$ & $.1 \mathrm{~km}$ dwn. Fire Crk. & LSP & & & & & 92 & 1.6 & NO MARK & $\mathrm{U}$ \\
\hline $7 / 28 / 2002$ & $.1 \mathrm{~km}$ dwn. Fire Crk. & LSP & & & & & 122 & 3.5 & NO MARK & $\mathrm{U}$ \\
\hline $7 / 28 / 2002$ & $.1 \mathrm{~km}$ dwn. Fire Crk. & LSP & & & & & 54 & 0.4 & NO MARK & $\mathrm{U}$ \\
\hline $7 / 28 / 2002$ & $.1 \mathrm{~km}$ dwn. Fire Crk. & LSP & & & & & 104 & 1.9 & NO MARK & $\mathrm{U}$ \\
\hline $7 / 28 / 2002$ & $.1 \mathrm{~km}$ dwn. Fire Crk. & LSP & & & & & 119 & 2.7 & NO MARK & $\mathrm{U}$ \\
\hline $7 / 28 / 2002$ & $.1 \mathrm{~km}$ dwn. Fire Crk. & LSP & & & & & 105 & 2.1 & NO MARK & $\mathrm{U}$ \\
\hline $7 / 28 / 2002$ & $.1 \mathrm{~km}$ dwn. Fire Crk. & LSP & & & & & 57 & 0.8 & NO MARK & $\mathrm{U}$ \\
\hline $7 / 28 / 2002$ & $.1 \mathrm{~km}$ dwn. Fire Crk. & LSP & & & & & 98 & 1.6 & NO MARK & $\mathrm{U}$ \\
\hline $7 / 28 / 2002$ & $.1 \mathrm{~km}$ dwn. Fire Crk. & LSP & & & & & 89 & 1.4 & NO MARK & $\mathrm{U}$ \\
\hline $7 / 28 / 2002$ & $.1 \mathrm{~km}$ dwn. Fire Crk. & LSP & & & & & 73 & 0.7 & NO MARK & $\mathrm{U}$ \\
\hline $7 / 28 / 2002$ & $.1 \mathrm{~km}$ dwn. Fire Crk. & LSP & & & & & 94 & 1.5 & NO MARK & $\mathrm{U}$ \\
\hline
\end{tabular}

1 RCO-Right center orange

2 T-transformed; U-untransformed 
Table A4. (con't).

\begin{tabular}{|c|c|c|c|c|c|c|c|c|c|c|}
\hline $\begin{array}{c}\text { Date } \\
\text { Electrofished }\end{array}$ & $\begin{array}{c}\mathrm{Km} / \\
\text { Reach }\end{array}$ & $\begin{array}{c}\text { Habitat } \\
\text { Type }\end{array}$ & $\begin{array}{c}\text { Area } \\
\text { Fished } \\
\left(\mathrm{m}^{2}\right) \\
\end{array}$ & $\begin{array}{c}\text { Time } \\
\text { Fished } \\
\text { (Min.) }\end{array}$ & $\begin{array}{l}\text { Lamprey } \\
\text { Captured }\end{array}$ & $\begin{array}{c}\text { Lamprey } \\
\text { Measured }\end{array}$ & $\begin{array}{c}\text { Length } \\
(\mathrm{mm})\end{array}$ & $\begin{array}{c}\text { Weight } \\
(\mathrm{g})\end{array}$ & Mark $^{1}$ & Phase $^{2}$ \\
\hline $7 / 28 / 2002$ & $.1 \mathrm{~km}$ dwn. Fire Crk. & LSP & & & & & 56 & 0.5 & NO MARK & $\mathrm{U}$ \\
\hline $7 / 28 / 2002$ & $.1 \mathrm{~km}$ dwn. Fire Crk. & LSP & & & & & 84 & 1.0 & NO MARK & $\mathrm{U}$ \\
\hline $7 / 28 / 2002$ & $.1 \mathrm{~km}$ dwn. Fire Crk. & LSP & & & & & 85 & 1.2 & NO MARK & $\mathrm{U}$ \\
\hline $7 / 28 / 2002$ & $.1 \mathrm{~km}$ dwn. Fire Crk. & LSP & & & & & 105 & 1.9 & NO MARK & $\mathrm{U}$ \\
\hline $7 / 28 / 2002$ & $.1 \mathrm{~km}$ dwn. Fire Crk. & LSP & & & & & 112 & 2.4 & NO MARK & $\mathrm{U}$ \\
\hline $7 / 28 / 2002$ & $.1 \mathrm{~km}$ dwn. Fire Crk. & LSP & & & & & 63 & -- & NO MARK & $\mathrm{U}$ \\
\hline $7 / 28 / 2002$ & $.1 \mathrm{~km}$ dwn. Fire Crk. & LSP & & & & & 67 & 0.8 & NO MARK & $\mathrm{U}$ \\
\hline $7 / 28 / 2002$ & $.1 \mathrm{~km}$ dwn. Fire Crk. & LSP & & & & & 77 & 0.9 & NO MARK & $\mathrm{U}$ \\
\hline $7 / 28 / 2002$ & $.1 \mathrm{~km}$ dwn. Fire Crk. & LSP & & & & & 71 & 0.9 & NO MARK & $\mathrm{U}$ \\
\hline $7 / 28 / 2002$ & $.1 \mathrm{~km}$ dwn. Fire Crk. & LSP & & & & & 51 & 0.2 & NO MARK & $\mathrm{U}$ \\
\hline $7 / 28 / 2002$ & $.1 \mathrm{~km}$ dwn. Fire Crk. & LSP & & & & & 45 & 0.2 & NO MARK & $\mathrm{U}$ \\
\hline $7 / 28 / 2002$ & $.1 \mathrm{~km}$ dwn. Fire Crk. & LSP & & & & & 74 & 0.8 & NO MARK & $\mathrm{U}$ \\
\hline $7 / 28 / 2002$ & $.1 \mathrm{~km}$ dwn. Fire Crk. & LSP & & & & & 52 & 0.3 & NO MARK & $\mathrm{U}$ \\
\hline $9 / 26 / 2002$ & $.1 \mathrm{~km}$ dwn. Fire Crk. & na & na & na & 6 & 6 & 39 & -- & NO MARK & $\mathrm{U}$ \\
\hline $9 / 26 / 2002$ & $.1 \mathrm{~km}$ dwn. Fire Crk. & na & & & & & 40 & -- & NO MARK & $\mathrm{U}$ \\
\hline $9 / 26 / 2002$ & $.1 \mathrm{~km}$ dwn. Fire Crk. & na & & & & & 40 & -- & NO MARK & $\mathrm{U}$ \\
\hline $9 / 26 / 2002$ & $.1 \mathrm{~km}$ dwn. Fire Crk. & na & & & & & 44 & -- & NO MARK & $\mathrm{U}$ \\
\hline $9 / 26 / 2002$ & $.1 \mathrm{~km}$ dwn. Fire Crk. & na & & & & & 39 & -- & NO MARK & $\mathrm{U}$ \\
\hline $9 / 26 / 2002$ & $.1 \mathrm{~km}$ dwn. Fire Crk. & na & & & & & 46 & -- & NO MARK & $\mathrm{U}$ \\
\hline
\end{tabular}

1. RCO - Right center orange

2. T - Transformed; U - Untransformed 
Table A5. Pacific lamprey presence-absence surveys in S.F. Clearwater River drainage, ID, 2002.

\begin{tabular}{|c|c|c|c|c|c|c|c|c|c|}
\hline \multirow[b]{2}{*}{ DATE } & \multirow[b]{2}{*}{ TIME } & \multirow[b]{2}{*}{ STREAM } & \multirow[b]{2}{*}{ RKM/DESCRIPT. } & \multicolumn{5}{|c|}{ E.FISHED } & \multirow[b]{2}{*}{$\begin{array}{c}\text { SHADE } \\
(\%) \\
\end{array}$} \\
\hline & & & & $\begin{array}{c}\text { AIR } \\
\text { TEMP }\left({ }^{0} \mathrm{C}\right) \\
\end{array}$ & $\begin{array}{c}\text { STREAM } \\
\text { TEMP }\left({ }^{0} \mathrm{C}\right) \\
\end{array}$ & $\begin{array}{l}\text { TIME } \\
(\min ) \\
\end{array}$ & $\begin{array}{l}\text { LAMPREY } \\
\text { CAPTURED }\end{array}$ & $\begin{array}{r}\text { DEPTH } \\
\text { AVE.(m) }\end{array}$ & \\
\hline $6 / 13 / 2002$ & 1120 & Am. R. & $.75 \mathrm{~km}$ abv. trlhd. 443 & na & 10.0 & 7.5 & 0 & 0.750 & not taken \\
\hline $6 / 13 / 2002$ & 1235 & Am. R. & $.37 \mathrm{~km}$ abv.trlhd. 443 & na & 12.0 & 5.0 & 0 & 0.363 & not taken \\
\hline $6 / 14 / 2002$ & 1050 & W. Fk. Am. R. & $.6 \mathrm{mi}$. blw. Table Mdw. & na & 6.0 & 9.0 & 0 & 0.288 & 100 \\
\hline $6 / 14 / 2002$ & 1328 & Am. R. & 100m S. of cmpgrd. & na & 14.0 & 8.0 & 0 & 0.538 & not taken \\
\hline $6 / 14 / 2002$ & 1421 & Am. R. & 2km USFS rd. 443 & na & 15.0 & 6.0 & 0 & 0.638 & not taken \\
\hline $6 / 14 / 2002$ & 1522 & Am. R. & $1 \mathrm{~km}$ blw. brdg. rd. 443 & na & na & 7.0 & 0 & 0.500 & not taken \\
\hline $6 / 15 / 2002$ & 1000 & Little Elk & $.1 \mathrm{~km}$ blw. rd. 686.1 & na & 10.0 & 6.0 & 0 & 0.325 & 100 \\
\hline $6 / 15 / 2002$ & 1155 & Am. R. & 50m blw. rd. 1189 brdg. & na & 14.0 & 8.0 & 0 & 0.375 & not taken \\
\hline $6 / 15 / 2002$ & 1404 & Am. R. & $1 \mathrm{~km}$ blw. rd. 1189 brdg. & na & 16.5 & 6.0 & 0 & 0.463 & not taken \\
\hline $6 / 15 / 2002$ & 1527 & Am. R. & 1km blw. Buffalo Gulch & na & 15.5 & 6.0 & 0 & 0.288 & not taken \\
\hline $7 / 2 / 2002$ & 1109 & Am. R. & $2 \mathrm{~km}$ up frm. mouth & 20.0 & 14.0 & 7.0 & 0 & 0.425 & not taken \\
\hline $8 / 8 / 2002$ & 915 & Am. R. & $.5 \mathrm{~km}$ up frm. mouth & 7.0 & 11.0 & 6.0 & 0 & 0.275 & not taken \\
\hline $9 / 5 / 2002$ & 1026 & S. F. C. R. & $.2 \mathrm{~km}$ abv. Cougar Crk. & 16.0 & 14.5 & 12.0 & 24 & $<1.00,>.200$ & not taken \\
\hline $9 / 6 / 2002$ & 950 & S. F. C. R. & $.3 \mathrm{~km}$ abv. Nlsn. Cmpgrd. & 14.5 & 14.0 & 13.0 & 50 & est. .400 & not taken \\
\hline
\end{tabular}


Table A6. Pacific lamprey presence-absence survey habitat descriptors in S.F. Clearwater River drainage, ID, 2002.

\begin{tabular}{|c|c|c|c|c|c|c|c|c|c|}
\hline \multirow[b]{2}{*}{ STREAM } & \multirow[b]{2}{*}{ RKM/DESCRIPT. } & \multirow{2}{*}{$\begin{array}{c}\text { E. FISHED } \\
\text { AREA } \\
\left(\mathrm{m}^{2}\right)\end{array}$} & \multirow[b]{2}{*}{ SILT/0RG. } & \multicolumn{3}{|c|}{ DOMINANT SUBSTRATE } & \multirow{2}{*}{\multicolumn{3}{|c|}{$\begin{array}{rrr}\text { SM. } & \text { LG. } \\
\text { BLDR. } & \text { BLDR. } & \text { OTHER }\end{array}$}} \\
\hline & & & & SAND & GRVL. & COBBLE & & & \\
\hline Am. R. & $.75 \mathrm{~km}$ abv. Trlhd. 443 & 5.0 & $3 *$ & & $4^{*}$ & $2^{*}$ & $1 *$ & & \\
\hline Am. R. & $.37 \mathrm{~km}$ abv.trlhd. 443 & 4.5 & $3 *$ & $1 *$ & $2 *$ & & & & \\
\hline W. Fk. Am. R. & .6mi. blw. Table Mdw. & 8.0 & & $1 *$ & & $2^{*}$ & & & \\
\hline Am. R. & 100m S. of cmpgrd. & 5.0 & & $1 *$ & $2^{*}$ & & & & \\
\hline Am. R. & 2km USFS rd. 443 & 5.0 & $1 *$ & & $2 *$ & & & & \\
\hline Am. R. & $1 \mathrm{~km}$ blw. brdg. rd. 443 & 5.0 & & & $1 *$ & $2^{*}$ & & & \\
\hline Little Elk & $.1 \mathrm{~km}$ blw. rd. 686.1 & 5.0 & $3 *$ & $1 *$ & $2 *$ & & & & \\
\hline Am. R. & 50m blw. rd. 1189 brdg. & 5.0 & & $1^{*}$ & & & & & \\
\hline Am. R. & $1 \mathrm{~km}$ blw. rd. 1189 brdg. & 5.0 & $2^{*}$ & $1^{*}$ & $2^{*}$ & & & & \\
\hline Am. R. & 1km blw. Buffalo Gulch & 5.0 & & $2^{*}$ & $1^{*}$ & $3^{*}$ & & & \\
\hline Am. R. & $2 \mathrm{~km}$ up frm. mouth & 5.0 & & $1^{*}$ & $3 *$ & $2^{*}$ & & & \\
\hline Am. R. & $.5 \mathrm{~km}$ up frm. mouth & 5.0 & $3 *$ & $2 *$ & $1 *$ & & & & \\
\hline S. F. C. R. & $.2 \mathrm{~km}$ abv. Cougar Crk. & 10.0 & 10 & 90 & & & & & \\
\hline S. F. C. R. & $.3 \mathrm{~km}$ abv. Nlsn. Cmpgrd. & 10.0 & 30 & & & 10 & 55 & & 5 \\
\hline
\end{tabular}

$1 *$ is the most abundant, $2 *$ being the second most abundant, etc. 
Table A7. Pacific lamprey presence-absence surveys in Selway River drainage, ID, 2002.

\begin{tabular}{|c|c|c|c|c|c|c|c|c|c|}
\hline \multirow[b]{2}{*}{ DATE } & \multirow[b]{2}{*}{ TIME } & \multirow[b]{2}{*}{ STREAM } & \multirow[b]{2}{*}{ RKM/DESCRIPT. } & \multicolumn{5}{|c|}{ E.FISHED } & \multirow[b]{2}{*}{$\begin{array}{c}\text { SHADE } \\
(\%)\end{array}$} \\
\hline & & & & $\begin{array}{c}\text { AIR } \\
\text { TEMP }\left({ }^{0} \mathrm{C}\right) \\
\end{array}$ & $\begin{array}{c}\text { STREAM } \\
\text { TEMP }\left({ }^{0} \mathrm{C}\right) \\
\end{array}$ & $\begin{array}{l}\text { TIME } \\
(\mathrm{min}) \\
\end{array}$ & $\begin{array}{l}\text { LAMPREY } \\
\text { CAPTURED }\end{array}$ & $\begin{array}{r}\text { DEPTH } \\
\text { AVE.(m) }\end{array}$ & \\
\hline $7 / 11 / 2002$ & 1044 & Whitecap Crk. & $1.5 \mathrm{~km}$ up mouth & 19.0 & 13.0 & 6 & 0 & 0.263 & not taken \\
\hline $7 / 11 / 2002$ & 1214 & Selway R. & 1.6km abv. Whitecap Crk. Brdg. & 25.0 & 16.0 & 6 & 0 & 0.363 & not taken \\
\hline $7 / 11 / 2002$ & 1442 & Selway R. & 10m above L.Clrwtr R. & 24.0 & 17.0 & 7 & 0 & 0.288 & not taken \\
\hline $7 / 12 / 2002$ & 1010 & Selway R. & 1.4km above L.Clrwtr R. & 32.0 & 14.0 & 6 & 0 & 0.350 & not taken \\
\hline $7 / 12 / 2002$ & 1122 & Deep Crk. & $1 \mathrm{~km}$ up frm. mouth & 23.0 & 15.0 & 7 & 0 & 0.250 & not taken \\
\hline $7 / 13 / 2002$ & 927 & Selway R. & 120 m dwn. Whitecap Crk. & 23.0 & 15.0 & 6 & 0 & 0.413 & not taken \\
\hline $7 / 13 / 2002$ & 1140 & Indian Crk. & $65 \mathrm{~m}$ up from mouth & 24.0 & 15.0 & 10 & 0 & 0.425 & not taken \\
\hline $7 / 13 / 2002$ & 1412 & Lit. Clrwtr R. & $140 \mathrm{~m}$ up from mouth & 23.0 & 15.0 & 7 & 0 & 0.588 & 100 \\
\hline $7 / 13 / 2002$ & 1624 & Selway R. & $1 \mathrm{~km}$ abv. Magruder R.S. & 23.0 & 16.0 & 6 & 0 & 0.263 & not taken \\
\hline $7 / 29 / 2002$ & 1010 & Meadow Crk. & $1 \mathrm{~km}$ up from mouth & 20.0 & 16.0 & 10 & 0 & 0.413 & not taken \\
\hline $7 / 29 / 2002$ & 1237 & Gedney Crk. & $50 \mathrm{~m}$ up from mouth & 16.0 & 14.0 & 8 & 0 & 0.325 & not taken \\
\hline 9/7/2002 & 1137 & Selway R. & 150m abv. Gedney Crk. & 15.0 & 16.5 & 16 & 16 & 0.238 & not taken \\
\hline $9 / 8 / 2002$ & 1113 & Selway R. & 100m abv. Jnsn. Bar & 17.5 & 17.0 & 13 & 45 & 0.238 & not taken \\
\hline $9 / 26 / 2002$ & na & Selway R. & Johnson Bar & -- & 15.0 & -- & 3 & -- & not taken \\
\hline
\end{tabular}


Table A8. Pacific lamprey presence-absence survey habitat descriptors in Selway River drainage, ID, 2002.

\begin{tabular}{|c|c|c|c|c|c|c|c|c|c|}
\hline STREAM & RKM/DESCRIPT. & $\begin{array}{c}\text { E. FISHED } \\
\text { AREA } \\
\left(\mathrm{m}^{2}\right) \\
\end{array}$ & SILT/0RG. & SAND & $\begin{array}{l}\text { DOMINANT } \\
\text { SUBSTRATE }\end{array}$ & COBBLE & $\begin{array}{c}\text { SM. } \\
\text { BLDR. }\end{array}$ & $\begin{array}{c}\text { LG. } \\
\text { BLDR. }\end{array}$ & OTHER \\
\hline WhiteCap Crk. & $1.5 \mathrm{~km}$ up mouth & 5.0 & & $3 *$ & & $2 *$ & $1 *$ & & \\
\hline Selway R. & 1.6km abv. WhiteCap Crk. Brdg. & 5.0 & $2 *$ & $3 *$ & $4 *$ & $1 *$ & $5 *$ & & \\
\hline Selway R. & 10m above L.Clrwtr R. & 5.0 & & $2 *$ & $3 *$ & $1 *$ & & & \\
\hline Selway R. & 1.4km above L.Clrwtr R. & 5.0 & $2 *$ & $1 *$ & $3 *$ & & & & \\
\hline Deep Crk. & $1 \mathrm{~km}$ up frm. mouth & 5.0 & & $2 *$ & & $1 *$ & $3 *$ & & \\
\hline Selway R. & 120m dwn. WhiteCap Crk. & 5.0 & $4^{*}$ & $1 *$ & $2 *$ & & & $3 *$ & \\
\hline Indian Crk. & $65 \mathrm{~m}$ up from mouth & 5.0 & $4^{*}$ & & $3^{*}$ & $2 *$ & $1 *$ & & \\
\hline Lit. Clrwtr R. & $140 \mathrm{~m}$ up from mouth & 5.0 & & & $3 *$ & $2 *$ & $1 *$ & $4 *$ & \\
\hline Selway R. & $1 \mathrm{~km}$ abv. Magruder R.S. & 5.0 & & $1^{*}$ & $2 *$ & & $3 *$ & & \\
\hline Meadow Crk. & $1 \mathrm{~km}$ up from mouth & 5.0 & $2^{*}$ & $1^{*}$ & & & $3 *$ & & \\
\hline Gedney Crk. & $50 \mathrm{~m}$ up from mouth & 5.0 & & $1 *$ & & $4 *$ & $2 *$ & $3 *$ & \\
\hline Selway R. & 150m abv. Gedney Crk. & 5.0 & & 20 & 10 & & 55 & 8 & 7 \\
\hline Selway R. & 100m abv. Jnsn. Bar & 5.0 & & 30 & & 10 & 45 & 5 & 10 \\
\hline Selway R. & Johnson Bar & -- & -- & -- & -- & -- & -- & -- & -- \\
\hline
\end{tabular}

$1 *$ is the most abundant, $2 *$ being the second most abundant, etc. 
Table A9. Pacific lamprey presence-absence surveys in Lochsa River drainage, ID, 2002.

\begin{tabular}{|c|c|c|c|c|c|c|c|c|c|}
\hline \multirow[b]{2}{*}{ DATE } & \multirow[b]{2}{*}{ TIME } & \multirow[b]{2}{*}{ STREAM } & \multirow[b]{2}{*}{ RKM/DESCRIPT. } & \multicolumn{5}{|c|}{ E.FISHED } & \multirow[b]{2}{*}{$\begin{array}{c}\text { SHADE } \\
(\%) \\
\end{array}$} \\
\hline & & & & $\begin{array}{c}\text { AIR } \\
\text { TEMP }\left({ }^{0} \mathrm{C}\right) \\
\end{array}$ & $\begin{array}{l}\text { STREAM } \\
\text { TEMP }\left({ }^{0} \mathrm{C}\right) \\
\end{array}$ & $\begin{array}{l}\text { TIME } \\
(\mathrm{min})\end{array}$ & $\begin{array}{l}\text { LAMPREY } \\
\text { CAPTURED }\end{array}$ & $\begin{array}{r}\text { DEPTH } \\
\text { AVE.(m) }\end{array}$ & \\
\hline $7 / 15 / 2002$ & 1010 & Crooked Fk. & 80m abv. Shtgn. Crk. & 23.0 & 11.0 & 8.5 & 0 & 0.388 & not taken \\
\hline $7 / 15 / 2002$ & 1128 & Shotgun Crk. & $80 \mathrm{~m}$ abv. Mouth & 19.0 & 11.0 & 5.5 & 0 & 0.175 & not taken \\
\hline $7 / 15 / 2002$ & 1354 & Brushy Fk. & Bvr. Rdg. L.O. Brdg. & 21.0 & 15.0 & 6.0 & 0 & 0.350 & not taken \\
\hline $7 / 16 / 2002$ & 919 & Lochsa R. & $1 \mathrm{~km}$ blw. Devoto Cdrs. & 16.0 & 13.0 & 9.0 & 0 & 0.300 & not taken \\
\hline $7 / 16 / 2002$ & 1208 & ColtKld. Crk. & $1.4 \mathrm{~km}$ abv. Mouth & 19.0 & 15.0 & 9.0 & 0 & 0.200 & not taken \\
\hline $7 / 16 / 2002$ & 1514 & Lochsa R & 3km blw. Pwll. R.S. & 27.0 & 17.0 & 6.0 & 0 & 0.263 & not taken \\
\hline $7 / 25 / 2002$ & 929 & Lochsa R. & Whthse. Cmpgrd. & 19.0 & 15.0 & 8.0 & 0 & 0.163 & not taken \\
\hline $7 / 25 / 2002$ & 1157 & Squaw Crk. & $.3 \mathrm{~km}$ abv. mouth & 25.0 & 14.0 & 5.0 & 0 & 0.300 & not taken \\
\hline $7 / 25 / 2002$ & 1403 & Lochsa R & 1.5km blw. Sqw. Crk. & 22.0 & 18.0 & 4.0 & 0 & 0.188 & not taken \\
\hline $7 / 26 / 2002$ & 957 & Lochsa R & $.2 \mathrm{~km}$ blw. mi. 146 & 22.0 & 18.0 & 8.0 & 0 & 0.313 & not taken \\
\hline $7 / 26 / 2002$ & 1201 & Lochsa R & $.2 \mathrm{~km}$ blw. Gngr. Crk. & 26.0 & 18.0 & 9.0 & 4 & 0.288 & not taken \\
\hline $7 / 27 / 2002$ & 936 & Lochsa R & $.1 \mathrm{~km}$ abv. Cstl. Crk. & 17.0 & 17.0 & 12.5 & 12 & 0.350 & not taken \\
\hline $7 / 28 / 2002$ & 1144 & Lochsa R & $.1 \mathrm{~km}$ blw. Fire Crk. & 25.0 & 17.5 & 11.0 & 38 & 0.388 & not taken \\
\hline $9 / 26 / 2002$ & na & Lochsa R & $.1 \mathrm{~km}$ Fire Crk. & na & 12.5 & na & 6 & na & not taken \\
\hline $7 / 30 / 2002$ & 1341 & P.O. Crk. & 80m abv. hwy.12 brdg. & 20.0 & 15.0 & 7.5 & 0 & 0.225 & not taken \\
\hline
\end{tabular}


Table A10. Pacific lamprey presence-absence survey habitat descriptors in Lochsa River drainage, ID, 2002.

\begin{tabular}{|c|c|c|c|c|c|c|c|c|c|}
\hline \multirow[b]{2}{*}{ STREAM } & \multirow[b]{2}{*}{ RKM/DESCRIPT. } & \multirow{2}{*}{$\begin{array}{c}\text { E. FISHED } \\
\text { AREA } \\
\left(\mathrm{m}^{2}\right) \\
\end{array}$} & \multicolumn{7}{|c|}{ DOMINANT SUBSTRATE } \\
\hline & & & SILT/0RG. & SAND & GRVL. & COBBLE & $\begin{array}{c}\text { SM. } \\
\text { BLDR. }\end{array}$ & $\begin{array}{c}\text { LG. } \\
\text { BLDR. }\end{array}$ & OTHER \\
\hline Crooked Fk. & 80m abv. Shtgn. Crk. & 5.0 & & $2 *$ & & $3 *$ & & $1 *$ & \\
\hline Shotgun Crk. & $80 \mathrm{~m}$ abv. Mouth & 1.0 & & & $3 *$ & $2 *$ & $1 *$ & & \\
\hline Brushy Fk. & Bvr. Rdg. L.O. Brdg. & 5.0 & & $2 *$ & & & $1 *$ & $3 *$ & \\
\hline Lochsa R. & $1 \mathrm{~km}$ blw. Devoto Cdrs. & 5.0 & $2 *$ & $1 *$ & $3 *$ & & $4 *$ & & \\
\hline ColtKld. Crk. & $1.4 \mathrm{~km}$ abv. Mouth & 5.0 & $1 *$ & $2 *$ & & & $3 *$ & $4 *$ & \\
\hline Lochsa R & 3km blw. Pwll. R.S. & 5.0 & & $2 *$ & & $1 *$ & $3 *$ & & \\
\hline Lochsa R. & Whthse. Cmpgrd. & 5.0 & $1 *$ & & & $3 *$ & $2 *$ & & \\
\hline Squaw Crk. & $.3 \mathrm{~km}$ abv. mouth & 5.0 & & $1 *$ & $3 *$ & $2 *$ & & & \\
\hline Lochsa R & $1.5 \mathrm{~km}$ blw. Sqw. Crk. & 1.0 & & & $3 *$ & $1 *$ & $2 *$ & & \\
\hline Lochsa R & $.2 \mathrm{~km}$ blw. mi. 146 & 5.0 & $4^{*}$ & $2 *$ & & $1 *$ & & & \\
\hline Lochsa R & $.2 \mathrm{~km}$ blw. Gngr. Crk. & 5.0 & $3 *$ & $3 *$ & & $1 *$ & $2 *$ & & \\
\hline Lochsa R & $.1 \mathrm{~km}$ abv. Cstl. Crk. & 5.0 & & $1 *$ & $2 *$ & & & $2 *$ & \\
\hline Lochsa R & $.1 \mathrm{~km}$ blw. Fire Crk. & est. 2.0 & $1 *$ & $2 *$ & & & & & \\
\hline Lochsa R & just blw. Fire Crk. & na & & & & & & & \\
\hline P.O. Crk. & $80 \mathrm{~m}$ abv. hwy. 12 brdg. & 5.0 & & & $1 *$ & $3 *$ & & $2 *$ & \\
\hline
\end{tabular}

$1 *$ is the most abundant, $2 *$ being the second most abundant, etc. 
Table A11. Pacific lamprey presence-absence surveys in Potlatch River drainage, ID, 2002.

\begin{tabular}{|c|c|c|c|c|c|c|c|c|c|}
\hline \multirow[b]{2}{*}{ DATE } & \multirow[b]{2}{*}{ TIME } & \multirow[b]{2}{*}{ STREAM } & \multirow[b]{2}{*}{ RKM/DESCRIPT. } & \multicolumn{5}{|c|}{ E.FISHED } & \multirow[b]{2}{*}{$\begin{array}{l}\text { SHADE } \\
(\%) \\
\end{array}$} \\
\hline & & & & $\begin{array}{c}\text { AIR } \\
\text { TEMP }\left({ }^{0} \mathrm{C}\right) \\
\end{array}$ & $\begin{array}{c}\text { STREAM } \\
\text { TEMP }\left({ }^{0} \mathrm{C}\right) \\
\end{array}$ & $\begin{array}{l}\text { TIME } \\
(\min )\end{array}$ & $\begin{array}{c}\text { LAMPREY } \\
\text { CAPTURED }\end{array}$ & $\begin{array}{c}\text { DEPTH } \\
\text { AVE.(m) }\end{array}$ & \\
\hline $6 / 20 / 2002$ & 1119 & Potlatch R. & 1mi. S. of Julietta & na & 13.0 & 5 & 0 & 0.350 & not taken \\
\hline $6 / 20 / 2002$ & 1257 & Potlatch R. & btwn Julietta \& Kendrick & na & 20.0 & 5 & 0 & 0.488 & not taken \\
\hline $6 / 20 / 2002$ & 1441 & Potlatch R. & $1.1 \mathrm{~km}$ E. of Kendrick & na & 22.0 & 5 & 0 & 0.638 & not taken \\
\hline $6 / 20 / 2002$ & 1704 & Potlatch R. & 80m blw. Rd. 1963 & na & 20.0 & 5 & 0 & 0.275 & not taken \\
\hline $6 / 20 / 2002$ & 1514 & Potlatch R. & Deary-Kendrick hwy. brdg. & na & 22.0 & 5 & 0 & est .200 & not taken \\
\hline $6 / 21 / 2002$ & 1144 & Potlatch R. & Ptltch. rd. brdg. E. of Kndrk. & na & 19.0 & 6 & 0 & 0.488 & not taken \\
\hline $6 / 24 / 2002$ & 1531 & Potlatch R. & Boulder Crk. Cmpgrd. & na & 22.0 & 4 & 0 & 0.275 & not taken \\
\hline $6 / 25 / 2002$ & 1432 & Big Bear Crk. & 2.5km blw. Mscw.-Dry. hwy. & 25.0 & 25.0 & 6 & 0 & 0.238 & not taken \\
\hline $6 / 26 / 2002$ & 1531 & E.F.Ptltch. R. & $2.5 \mathrm{~km}$ abv. Mouth & 30.0 & 24.0 & 7 & 0 & 0.250 & not taken \\
\hline $6 / 26 / 2002$ & 1322 & E.F.Ptltch. R. & abv. Rd. 4707 & 30.0 & 18.0 & 5 & 0 & 0.350 & not taken \\
\hline $6 / 27 / 2002$ & 1132 & Potlatch R. & $2.5 \mathrm{~km}$ abv. E. Fork & 27.0 & 24.0 & 6 & 0 & 0.488 & not taken \\
\hline $6 / 27 / 2002$ & 1340 & Potlatch R. & 60m abv. Moose Crk. & 27.0 & 23.0 & 5 & 0 & 0.475 & not taken \\
\hline $6 / 27 / 2002$ & 1443 & Potlatch R. & 1.5km abv. Jackson Crk. & 27.0 & 20.0 & 7 & 0 & 0.438 & not taken \\
\hline $7 / 1 / 2002$ & 1224 & Potlatch R. & 1.5km blw. Pine Crk. & 23.0 & 19.0 & 10 & 0 & 0.375 & not taken \\
\hline $7 / 1 / 2002$ & 1544 & Potlatch R. & $.4 \mathrm{~km}$ blw. Juliaetta & 23.0 & 24.0 & 7 & 0 & 0.450 & not taken \\
\hline
\end{tabular}




\section{Table A12. Pacific lamprey presence-absence survey habitat descriptors in Potlatch}

River drainage, ID, 2002.

\begin{tabular}{|c|c|c|c|c|c|c|c|c|c|}
\hline \multirow[b]{2}{*}{ STREAM } & \multirow[b]{2}{*}{ RKM/DESCRIPT. } & \multirow{2}{*}{$\begin{array}{c}\text { E. FISHED } \\
\text { AREA } \\
\left(\mathrm{m}^{2}\right) \\
\end{array}$} & \multirow[b]{2}{*}{ SILT/0RG. } & \multicolumn{5}{|c|}{ DOMINANT SUBSTRATE } & \multirow[b]{2}{*}{ OTHER } \\
\hline & & & & SAND & GRVL. & COBBLE & $\begin{array}{c}\text { SM. } \\
\text { BLDR. }\end{array}$ & $\begin{array}{c}\text { LG. } \\
\text { BLDR. }\end{array}$ & \\
\hline Potlatch R. & 1mi. S. of Julietta & 5.0 & $3^{*}$ & & $4^{*}$ & $2^{*}$ & $1^{*}$ & & \\
\hline Potlatch R. & btwn Julietta \& Kendrick & 10.0 & & $4^{*}$ & & $1 *$ & $3^{*}$ & $2^{*}$ & \\
\hline Potlatch R. & $1.1 \mathrm{~km}$ E. of Kendrick & 5.0 & & $2^{*}$ & $1 *$ & & & & \\
\hline Potlatch R. & 80m blw. Rd. 1963 & 5.0 & $3 *$ & & $4 *$ & $2^{*}$ & $1^{*}$ & & \\
\hline Potlatch R. & Deary-Kendrick hwy. brdg. & est. 5.0 & & & & & $1^{*}$ & & \\
\hline Potlatch R. & Ptltch. rd. brdg. E. of Kndrk. & 5.0 & $3^{*}$ & & & & $2^{*}$ & $1 *$ & \\
\hline Potlatch R. & Boulder Crk. Cmpgrd. & 5.0 & $2^{*}$ & $1 *$ & & & & & \\
\hline Big Bear Crk. & $2.5 \mathrm{~km}$ blw. Mscw.-Dry. hwy. & 5.0 & $3 *$ & & $4 *$ & $2 *$ & $1^{*}$ & & \\
\hline E.F.Ptltch. R. & $2.5 \mathrm{~km}$ abv. Mouth & 5.0 & $1^{*}$ & & $3 *$ & $2^{*}$ & & & \\
\hline E.F.Ptltch. R. & Abv. Rd. 4707 & est. 5.0 & $1 *$ & & & & & & \\
\hline Potlatch R. & $2.5 \mathrm{~km}$ abv. E. Fork & 5.0 & $4^{*}$ & & $3^{*}$ & $2^{*}$ & $1 *$ & & \\
\hline Potlatch R. & 60m abv. Moose Crk. & 5.0 & $2^{*}$ & $3^{*}$ & $1^{*}$ & & & & \\
\hline Potlatch R. & $1.5 \mathrm{~km}$ abv. Jackson Crk. & 5.0 & $2^{*}$ & $3^{*}$ & $1 *$ & $4^{*}$ & & & \\
\hline Potlatch R. & 1.5km blw. Pine Crk. & 10.0 & & & $2^{*}$ & $1 *$ & & & \\
\hline Potlatch R. & $.4 \mathrm{~km}$ blw. Juliaetta & 5.0 & & & & $3 *$ & $2^{*}$ & $1 *$ & \\
\hline
\end{tabular}

$1 *$ is the most abundant, $2 *$ being the second most abundant, etc. 
Table A13. Habitat descriptors at locations of Pacific lamprey habitat sampling in Red River, ID, 2002.

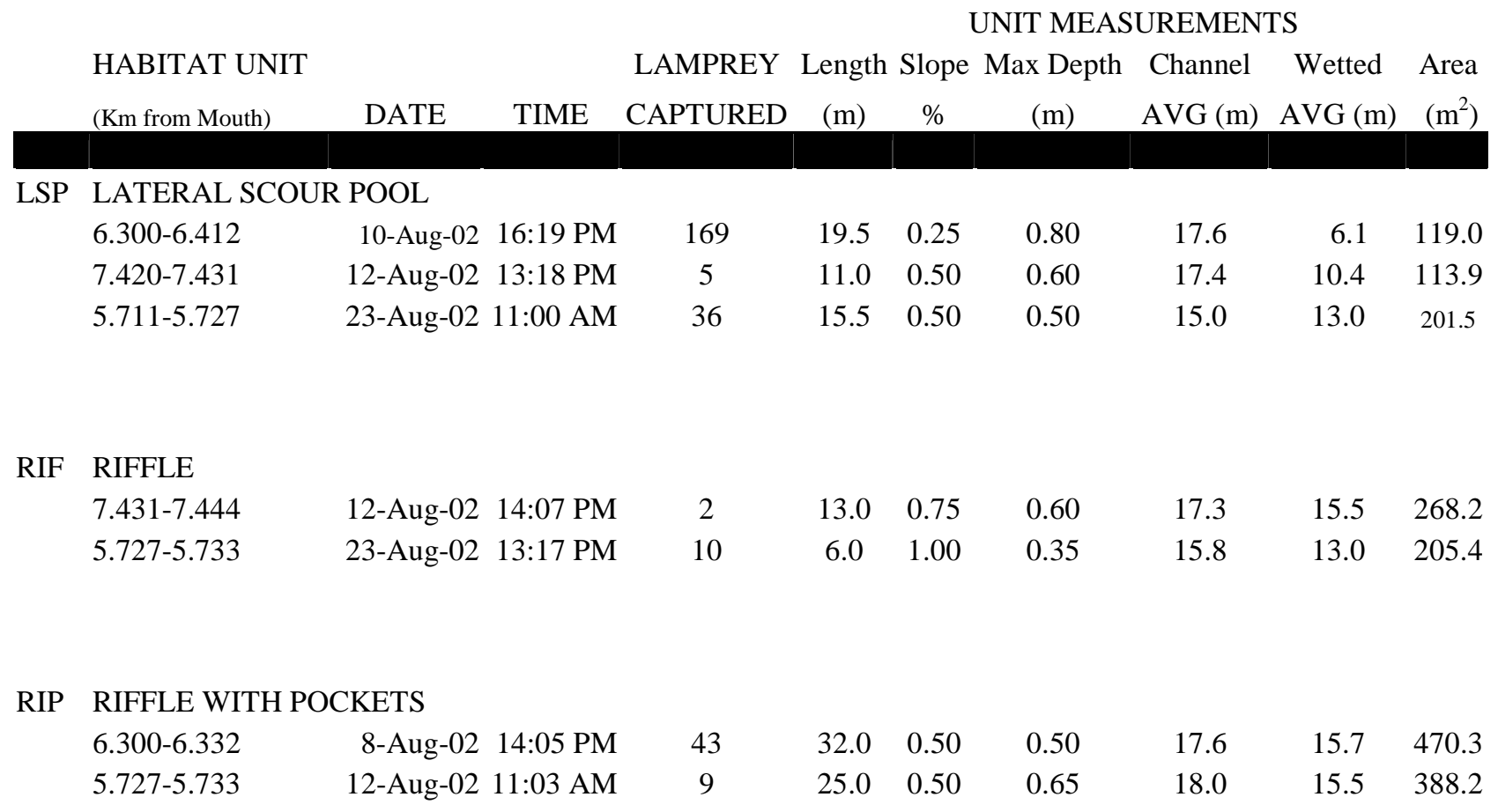


Table A14. Channel descriptions for habitat units surveyed for Pacific lamprey in Red River, ID, 2002.

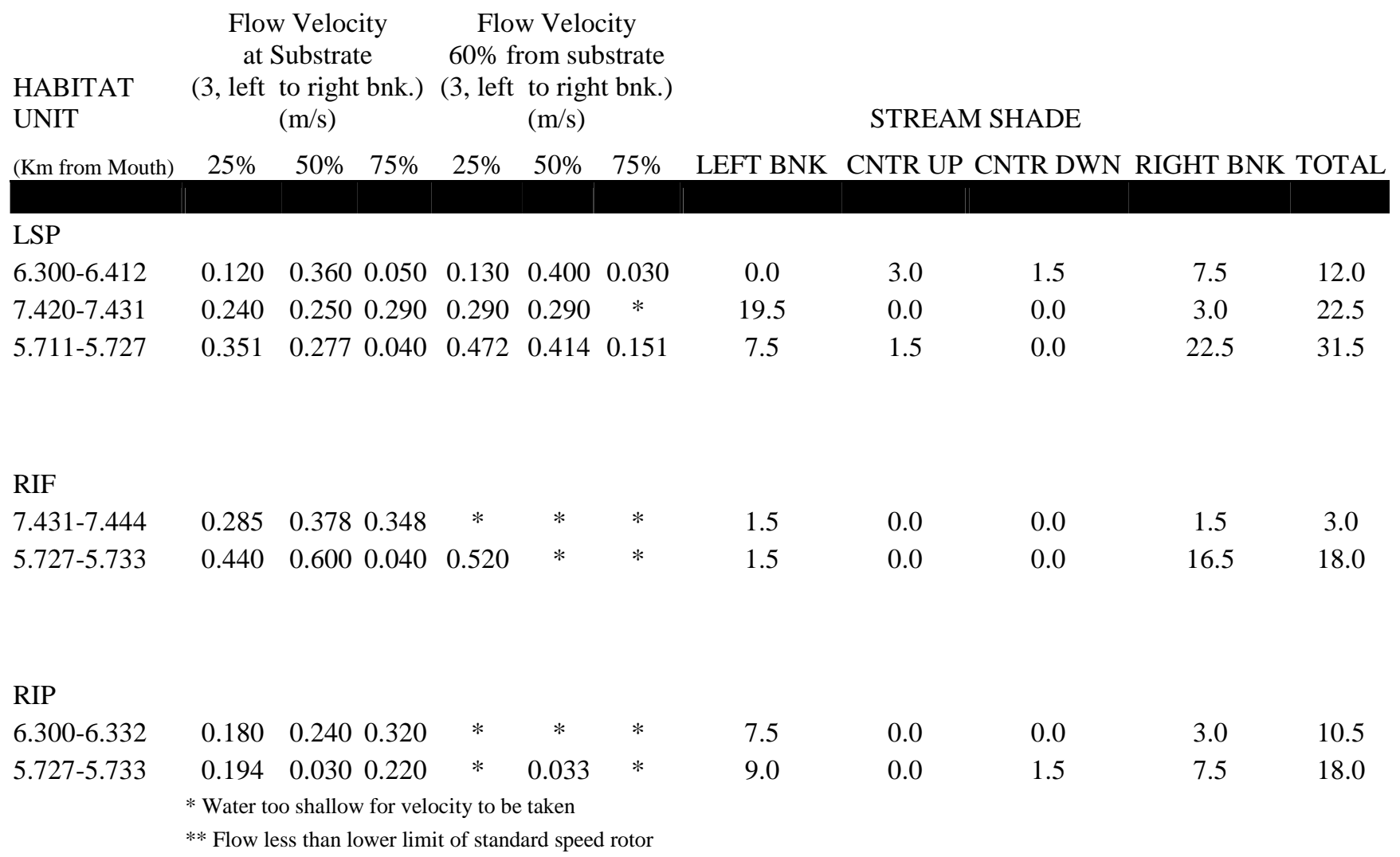


Table A15. Stream substrate descriptions at habitat unit sample sites in Red River, ID, 2002.

\begin{tabular}{|c|c|c|c|c|c|c|c|c|c|}
\hline \multirow{3}{*}{$\begin{array}{l}\text { HABITAT UNIT } \\
\text { (Km from Mouth) }\end{array}$} & \multicolumn{9}{|c|}{ SUBSTRATE COMPOSITION \% } \\
\hline & LG. & SM. & & COARSE & MED. & FINE & COARSE & FINE & SILT/ BED \\
\hline & BLDR & BLDR & COBBLE & GRVL. & GRVL & GRVL & SAND & SAND & ORG. ROCK \\
\hline & & & & & & & & & \\
\hline \multicolumn{10}{|l|}{ LSP } \\
\hline $6.300-6.412$ & 0.5 & 6.5 & 39.0 & 12.0 & 9.0 & 6.0 & 11.0 & 14.0 & 2.0 \\
\hline $7.420-7.431$ & 11.0 & 15.0 & 38.0 & 16.0 & 6.0 & 6.0 & 4.0 & 2.0 & 2.0 \\
\hline $5.711-5.727$ & 3.0 & 23.0 & 33.0 & 16.0 & 6.0 & 6.0 & 5.0 & 4.0 & 4.0 \\
\hline \multicolumn{10}{|l|}{ RIF } \\
\hline 7.431-7.444 & 4.0 & 9.0 & 31.0 & 15.0 & 11.0 & 8.0 & 11.0 & 5.0 & 6.0 \\
\hline $5.727-5.733$ & 3.0 & 7.0 & 38.0 & 17.0 & 11.0 & 9.0 & 8.0 & 5.0 & 2.0 \\
\hline \multicolumn{10}{|l|}{ RIP } \\
\hline $6.300-6.332$ & 7.0 & 10.0 & 46.0 & 13.0 & 6.0 & 5.0 & 5.0 & 3.0 & 5.0 \\
\hline $5.727-5.733$ & 5.0 & 7.5 & 46.0 & 15.0 & 7.0 & 7.0 & 6.0 & 4.0 & 2.5 \\
\hline
\end{tabular}

